\title{
The salmonid myostatin gene family: a novel model for investigating mechanisms that influence duplicate gene fate
}

\author{
Casey B Lawson ${ }^{1,3}$, Takumu Niino ${ }^{1,3}$, Russell A Hermansen ${ }^{4}$, Vera Brok-Volchanskaya ${ }^{4}$, Melissa F Jackson ${ }^{2,3}$,
} Dilip K Garikipati ${ }^{1,3}$, David A Liberles ${ }^{4^{*}}$ and Buel D Rodgers ${ }^{1,2,3^{*}}$

\begin{abstract}
Background: Most fishes possess two paralogs for myostatin, a muscle growth inhibitor, while salmonids are presumed to have four: mstn $1 a, m s t n 1 b$, mstn $2 a$ and $m s t n 2 b$, a pseudogene. The mechanisms responsible for preserving these duplicates as well as the depth of mstn $2 b$ nonfunctionalization within the family remain unknown. We therefore characterized several genomic clones in order to better define species and gene phylogenies.

Results: Gene organization and sequence conservation was particularly evident among paralog groupings and within salmonid subfamilies. All mstn2b sequences included in-frame stop codons, confirming its nonfunctionalization across taxa, although the indels and polymorphisms responsible often differed. For example, the specific indels within the Onchorhynchus tshawytscha and O. nerka genes were remarkably similar and differed equally from other mstn $2 b$ orthologs. A phylogenetic analysis weakly established a mstn $2 b$ clade including only these species, which coupled with a shared 51 base pair deletion might suggest a history involving hybridization or a shared phylogenetic history. Furthermore, mstn2 introns all lacked conserved splice site motifs, suggesting that the tissue-specific processing of mstn2a transcripts, but not those of mstn2b, is due to alternative cis regulation and is likely a common feature in salmonids. It also suggests that limited transcript processing may have contributed to mstn2b nonfunctionalization.

Conclusions: Previous studies revealed divergence within gene promoters while the current studies provide evidence for relaxed or positive selection in some coding sequence lineages. These results together suggest that the salmonid myostatin gene family is a novel resource for investigating mechanisms that regulate duplicate gene fate as paralog specific differences in gene expression, transcript processing and protein structure are all suggestive of active divergence.
\end{abstract}

Keywords: Salmon, Myostatin, Gene duplication, Subfunctionalization

\section{Background}

The manipulation of striated muscle size and growth has several potential applications in agriculture and medicine [1]. Such advances could help treat patients with muscular dystrophy, cancer cachexia, age-related sarcopenia and/or heart failure and in addition, improve

\footnotetext{
*Correspondence: liberles@uwyo.edu; danrodgers@wsu.edu

${ }^{4}$ Department of Molecular Biology, University of Wyoming, Laramie, WY 82071, USA

'Department of Animal Sciences, Washington State University, Pullman, WA 99164, USA

Full list of author information is available at the end of the article
}

livestock production [2-4]. Many developing technologies, those that either actively enhance striated muscle growth or those that screen for polymorphisms associated with enhanced growth, target myostatin; a potential endocrine as well as local inhibitor $[5,6]$. Indeed, attenuating myostatin experimentally creates a "double muscling" phenotype that also occurs in mstn-/- animals and in those overexpressing dominant-negative receptors or one of several known myostatin binding proteins [1].

The fundamental mechanisms of myostatin action in mammals are well known, but have only recently been described in other vertebrates, particularly fish [1]. The

\section{Biomed Central}


myokine appears to inhibit muscle progenitor cell proliferation in all systems, although studies with mammalian cell lines and primary fish myosatellite cells suggest it either inhibits or stimulates differentiation, respectively [7-11]. This discrepancy is partially explained by culture conditions and by the immortalized phenotype of cell lines. Nevertheless, it is one of several ways that myostatin biology differs between mammals and fish.

In fact, most fish species possess two distinct myostatin genes $[12,13]$ that were retained after an early genome duplication, specifically in ray-finned (Actinopterygii) fishes, over $300 \mathrm{Ma}$ ago $[14,15]$. The more recent tetraploidization of modern salmonids, approximately 25-100 Ma ago, produced four myostatin paralogs (mstn1a, mstn1b, $m s t n 2 a \& m s t n 2 b$ ), although $m s t n 2 b$ is a pseudogene in rainbow trout [16]. Each paralog is differentially expressed in rainbow trout and the mstn 2 transcripts are alternatively processed in a way that contributes to the nonfunctionalization of $m s t n 2 b$ and to the tissue-specific actions of mstn2a. The differential patterns of gene expression and transcript processing among the rainbow trout MSTN paralogs suggests that subfunctionalization (complementary \& compartmentalized function), neofunctionalization (adopting new functions) and nonfunctionalization may have all contributed to this gene family's evolution. A better understanding of myostatin gene structure and phylogenies among other salmonids would therefore help explain fundamental mechanisms that influence duplicate gene fate and ultimately control their fixation and maintenance, which in turn enables the functional diversification of genes and genomes [17].

The evolution of myostatin has previously been studied among mammalian orthologs, where it was found to be broadly conserved, except for periods of rapid sequence evolution in ruminant Artiodactyls [18]. Because of the suggested link between gene duplication and functional divergence, myostatin was studied within the salmonids. Reported herein is the structural characterization and phylogenetic analysis of 33 MSTN paralogs cloned from several species within the three salmonid subfamilies: Salmoninae, Coregonidae and Thymallidae, with Retropinna as an outgroup species. The results together describe a unique and novel gene family model for examining not only salmonid phylogenies, but also different molecular mechanisms that influence duplicate gene fate.

\section{Results}

\section{Comparative Mapping and Gene Organization}

The basic organization of each salmonid myostatin para$\log$ is highly conserved as each gene contains three exons (Figure 1). This is true not only in salmonids, but in all other vertebrates as well [1]. Gene structures are most similar among the mstn 1 genes, as indicated by conserved exon lengths, although the $m s t n 2 a$ genes differed in length

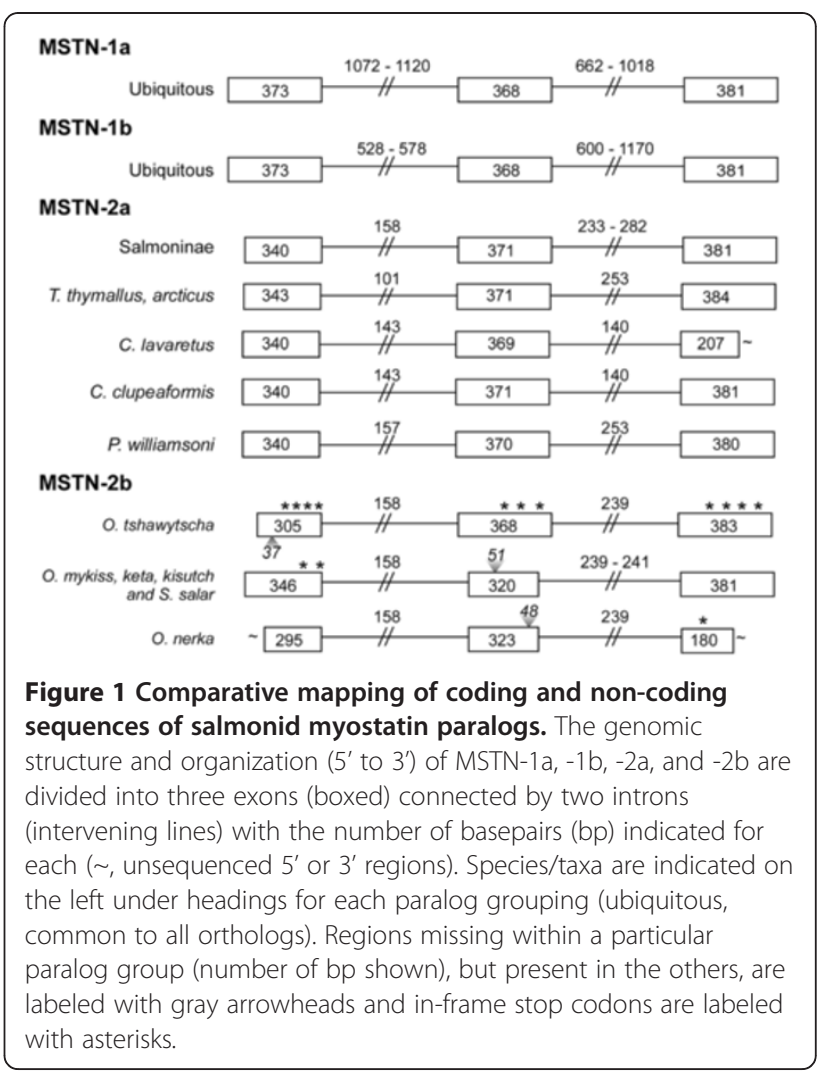

by only $1-3 \mathrm{bp}$. Most variability understandably occurred within introns and these differences were reflected in the taxa, particularly among the $m s t n 2 a$ genes. Intron sizes were also hierarchal as in general, mstn1a introns were largest followed by those of $m s t n 1 b$ and the $m s t n 2$ genes. Thus, differences in intron and exon size alone can often be used to distinguish individual paralogs, even if not computing molecular phylogenies.

$M s t n 2 b$ paralogs were only cloned from species within the Salmoninae subfamily and in every case, each was a pseudogene. Nonfunctionalization appears to have arisen independently among these genes as the in-frame stop codons occurred in different locals. This could also be due to mutations that occurred after nonfunctionalization, although a closer examination of the specific indels suggests this is not the case (see below). Three notable deletions include $37 \mathrm{bp}$ from the $O$. tshawytscha first exon, $48 \mathrm{bp}$ from the $O$. nerka second exon and $51 \mathrm{bp}$ from the O. mykiss, O. keta, O. kisutch and S. salar second exons. The fact that the latter 51 bp region is missing in S. salar and is retained in O. tshawytscha and $O$. nerka suggests that a common ancestor to these latter two species diverged well before the more recent salmonid radiation. This is supported by the similar distribution of stop codons, and underlying molecular changes (see below), as well as the retention of the aforementioned 17 and 48 bp regions in several other 
Oncorhynchus species and in S. salar. Pseudogenes evolve randomly and are not influenced by selection pressures at the protein or expression levels. Therefore, the signal in these pseudogenes will not convolute ancestral and functional (sometimes convergent) signal as is prone to happen in gene family evolution.

\section{Multiple sequence alignments}

The coding region for each orthologous group is well conserved as individual comparison between any two salmonid species indicated that the MSTN-1a, $-1 \mathrm{~b}$ and -2a proteins are on average $99 \%, 98 \%$ and $92 \%$ identical, respectively (Figures 2, 3, and 4). Several taxa-specific features were identified and could aid in determining gene family phylogenies. These include F262 that occurs in all Oncorhynchus MSTN-1a proteins (Figure 3). This substitution is particularly noteworthy as it lies within the furin/prohormone convertase (PC) recognition sequence that is necessary for the cleavage and formation of mature myostatin peptide [1]. In addition, V243 is common only to the Coregonid MSTN-1b sequences (Figure 3) and 27 unique positions were identified among the different MSTN-2a sequences (Figure 4). This includes a 7 bp deletion in exon 1 of Sv. fontinalis $m s t n 2 a$ that produces a frame shift, although an alternative upstream initiator results in a complete open reading frame that is very similar to the other MSTN-2a amino acid sequences (Figure 4). This pattern of conservation and divergence is reminiscent to that of intron sizes as it reflects a hierarchy where the coding sequences are most conserved among MSTN-1 paralogs and most divergent among the MSTN-2a.

Comparing the MSTN-2b cDNA sequences indicated, as with the gene organization data, that the $O$. tshawytscha and $O$. nerka genes are most similar. Each lack a 4 bp region at position 215-218 that is common to the other Oncorhynchus species and to S. salar (Figure 5). They also share a $5 \mathrm{bp}$ insertion at position 468-472 and an adjacent downstream region of $73 \mathrm{bp}$ that differs significantly from the other orthologs in addition to several other single nucleotide polymorphisms. Furthermore, the $O$. tshawytscha sequence lacks a $37 \mathrm{bp}$ cassette at position 132-169 and contains an additional 46 bp between 557-596. The former shifts the coding frame and is responsible for producing many stop codons (Figure 1). In all other sequences, excluding $O$. nerka, a single bp is missing at position 160, which introduces a frame shift and several premature stop codons. With an intact 1st exon coding region (e.g. no 37 bp deletion), the $O$. nerka coding frame changes multiple times before introducing a stop codon in the 3rd exon that is located after the region coding for the furin/ PC site (data not shown). This opens the possibility that a truncated and mutated myostatin prodomain (a.k.a. latency associated protein, LAP) for MSTN-2b could be produced in $O$. nerka. An assessment of motifs necessary for mRNA processing, however, suggests that this does not occur (see below). The similarities noted between $O$. tshawytscha and O. nerka sequences are together suggestive of a common ancestor that diverged early from other salmonids. The notable differences, however, likely occurred after the two species subsequently diverged from the ancestor. Nevertheless, these data could prove useful in reevaluating salmonid phylogenetic relationships.

\section{In silico assessment of mRNA splice site motifs}

Rainbow trout $m s t n 2$ transcripts are alternatively spliced in a manner that prevents the production of mature $m s t n 2 b$ transcripts in all tissues and limits mstn $2 a$ processing to specific tissues and developing conditions $[10,11,16]$. We therefore assessed exon/intron boundaries and identified putative branch points in each $m s t n 2$ gene to determine the likelihood of transcript processing across taxa. None of the boundaries, regardless of gene, possessed an intact splice site (Figure 6). At least one of the nucleotides flanking each boundary, those most critical for splicing, were mutated in all genes except for the 5 ' boundary of the second introns. Putative branch point motifs were identified in the first introns of all genes, although by contrast, these motifs were either missing or mutated in the second introns (Table 1). These data strongly suggest that the alternative processing of $m s t n 2$ transcripts is a common feature among Salmoninae species if not all salmonids. The boundary sequences were remarkably similar in all genes indicating that the underlying changes occurred in a basal salmonid species, but after tetraploidization as all $m s t n 1$ transcript processing sites are functionally intact. Thus, alterations in transcript processing may have precluded changes in coding sequences that together contributed to the divergence of $m s t n 2 a$ and nonfunctionalization of $m s t n 2 b$.

\section{Phylogenetic analysis}

Phylogenetic analysis of the myostatin genes revealed that the evolution of mstn1a and mstn $1 b$ closely resembles the established species phylogenies. The only exception was the grouping of $O$. kisutch mstn1b with $S$. fontinalis instead of other Oncorhynchus species (Figure 7). This exception was strengthened in the mstn1a tree estimated through MrBayes, which showed $O$. kisutch as the first genus to diverge and therefore might be the oldest member of the clade. The Oncorhynchus clade in the amino acid tree was poorly resolved by Phyml and little inference could be gained, however O. kisutch was placed as an outgroup to the clade by the ML method when using the DNA sequences with high bootstrap support. This relationship was strongly 


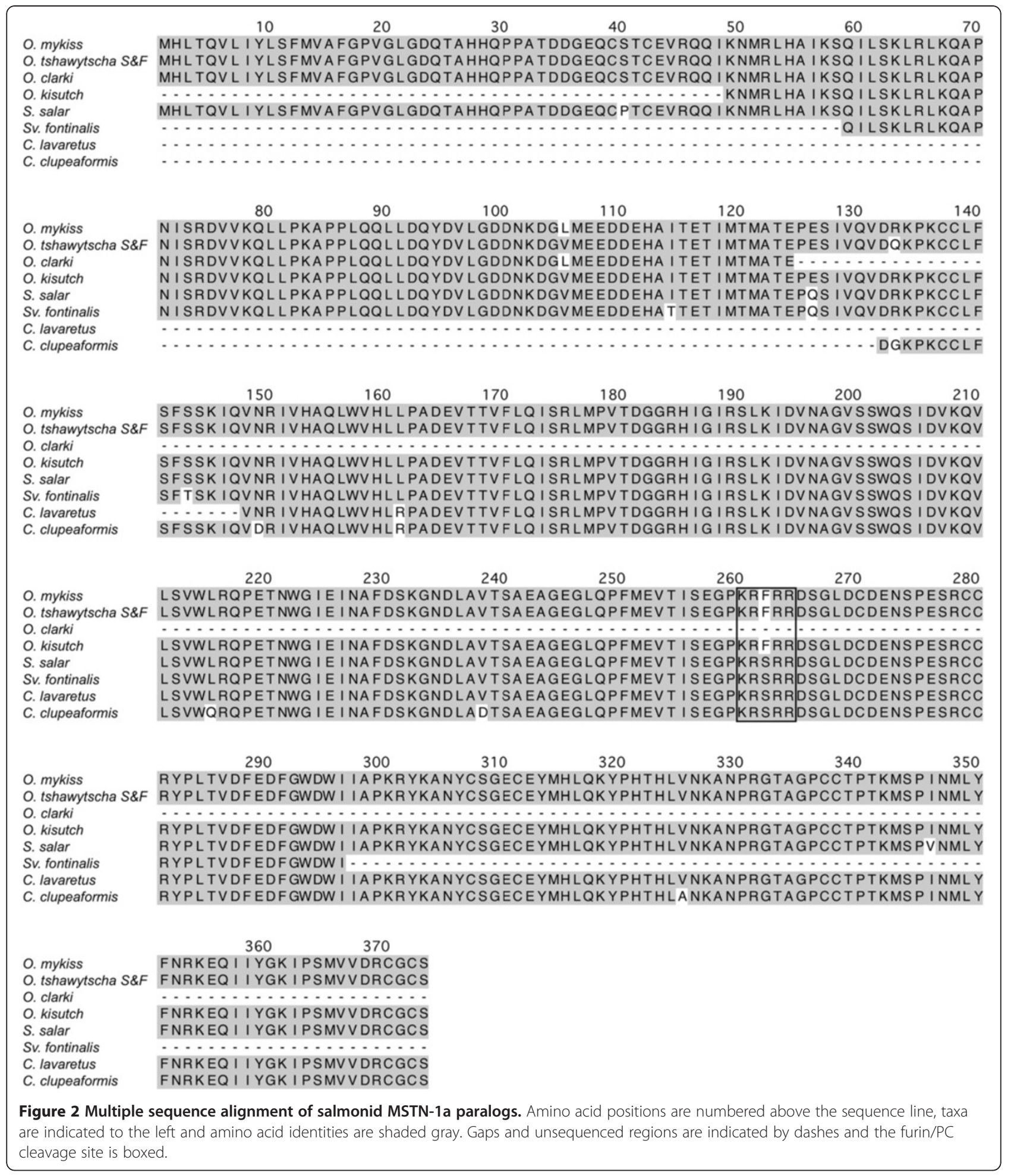

conserved in all of the trees for $m s t n 1 b$, produced through either a ML or Bayesian estimate. The mstn2 (Figure 8) phylogenies revealed more about the possible relationships between salmonids and in general, they appear to have diverged in accordance with the believed species tree, although with two notable exceptions.
Firstly, O. kisutch seems to have experienced more change than the rest of Oncorhynchus as demonstrated by the placement of $O$. kisutch mstn $2 a$ outside of the other Oncorhynchus mstn2a orthologs (Figure 8). This again suggests that it diverged earlier and has had more time to evolve. Secondly, there was weak support for an 


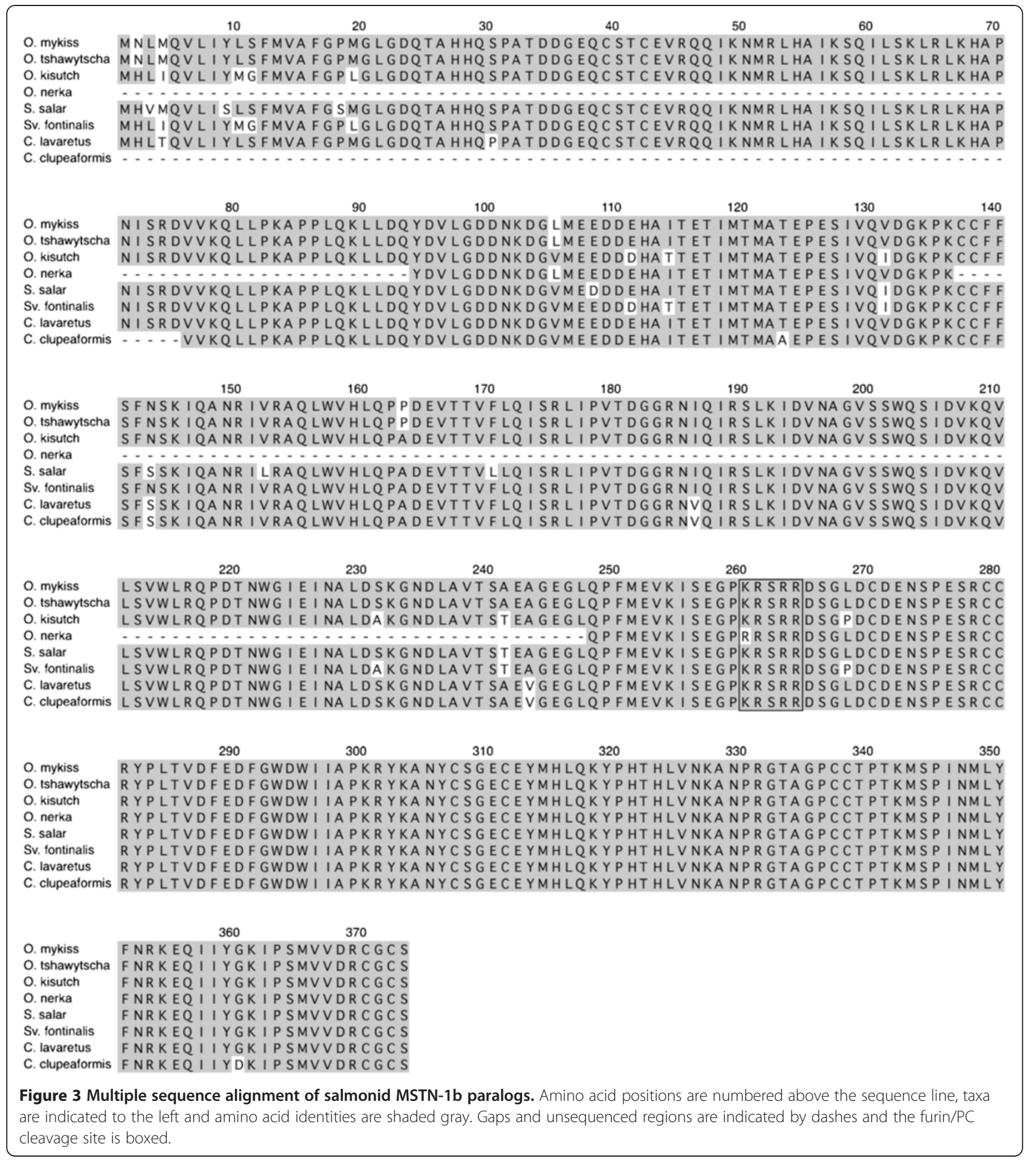

O. tshawytscha and O. nerka clade in the $m s t n 2 b$ tree at the DNA level. The mstn $1 b$ and mstn $2 a$ trees, by contrast, suggest evidence of an older common ancestor that separates the two taxa. Nevertheless, there was stronger support at the amino acid level as trees for MSTN-1b, MSTN-2a and MSTN-2b (Additional files 1 $\& 2$ ) indicate the relationship between $O$. tshawytscha and O. nerka is upheld with bootstrap support. Further, signal in the substitution data is complemented by the observation of shared rare indel events that are unlikely to have occurred independently by chance. A complete tree showing all MSTN1 and MSTN2 genes was generated using ML and Bayesian methods and can be seen in the supplementary data (Additional file 3). 
O. mykiss MQFMLYLTLLGVLSTTMGMNKTTRRQANVT-EEGEVQQCSNCEFREQSRLMRLHNIRSQILSILRLEQAP

O. tshawytscha MQFMLYLTLLGVLSTTMGMNETTRRQANVT-EEGEVQQCSNCEFREQSRLMRLHNIRSQILSILRLEQAP

O. clarki MQFMLYLTLLGVLSTTMGMNETTRRQANVT-EEGEVQQCSNCEFREQSRLMRLHNIRSQILSILRLEQAP

0. kisutch MQFMLYLTLLGVLSTTMGMNETTRRQANVT-EEGEVQQCSNCEFREQSRLMRLHNIRSQILSILRLEQAP

O. nerka MQFMLYLTLLGVLSTTMGMNETTRRQANVT-EEGEVQQCSNCEFREQSRLMRLHNIRSQILSILRLEQAP

S. salar MQFMLYLTLFGVLGTTMGMNETTRRLANVTTEEGEVQQCSNCEFREQSRQMRLHNIRSQILSILRLEQAP

Sv. Iontinalis
C. lavaretus

C. clupeatormis MQGMLYLTLLAALG-TMG INETTRRQANVTTVEGEGEQCSTCEFREQSRQMRLHNIRSQILSILRLEQAP

T. arcticus MQFLLYLTLLGALGTTMGMNETTRRQANVTTVEVEGEQCSTCEFREQSRLMRLHNIRSQILSILRLEQAP

T. thylamus MOFLLYLTLLGALGTTMGMNETTRROANVTTVEVEGEQCSTCEFREQSRLMRLHNIRSOILSILRLEQAP

P. williamsoni MQVMLYLTLLAALG-TMGINETTRRQANVTTVEGEGEQCSTCEFREQSRLMRLHNIRSQILSILRLEQAP

90

100

110

120

130

140

O. mykiss NISREMIRQLLPKAPPLTQLIDQYEHRVEDEERATTET I ITMAKPGPMSQQDG I PSCCFFNLSPKIRPNN

O. tshawytscha N I SREMIRQLLPKAPPLTQLI DQYEHRVEDEERATTET I I TMAKPGPMSQQDGIPSCCFFNLSPKIRPNN

O. clarki NISREMIRQLLPKAPPLTQLIDQYEHRVEDEERATTET I ITMAKPGPMSQQDGIPSCCFFNLSPKIRPNN

O. kisutch NISREMIRQLLPKAPPLTQLIDQYEHRVEDEERATTETIITMAKPGPMSOQDGIPSCCFFNLSPKIRPNN

O. nerka NISREMIROLLPKAPPLTQFIDQYEHRVEDEERATTETIITMAKPGPMSOQDGIPSCCFFNLSPKIRPNN

S. salar NISREMIRQLLPKAPPLTQLIDQYEHRVEDEERATTET I ITMAKPGPMSQQEGIPSCCFFNLSPKIRPNN

Sv. tontinalis NISGEMIRQLLPKAPILTQYIDQYEHRVEDEERATTET I ITMAKPGPMSQQDGIPSCCFFNLSPKIRPNN

C. lavaretus NISRDMIMQLLPKAPPLTQLMDQYEHRVEDEERATTETIITMAKPGPMSQEDGIPSCCFFNLSPKIRPNN

C. clupeatormis N I SRDM IMQLLPKAPPLTQLMDQYEHRVEDEERATTET I ITMAKPGPMSQEDG IPSCCFFNLSPK IRPNN

T. arcticus NISRDMIKQLIPKAPPLTQLMDQYEHRVEDEERATTET I ITMAKPGPMSQQDGIPSCCFFNLSPKIRPNN

T. thylamus NISRDMIKQLIPKAPPLTQLMDQYEHRVEDEERATTET I ITMAKPGPMSQQDGIPSCCFFNLSPKIRPNN

P. williansoni NISRDMIRQLLPKAPPLTQLMEQYEHRVEDEERATTETIITMAKPEPMSQEDGIPSCCFFNLSPKIRPNN

150

160

170

180

190

200

210

O. mykiss I LHAQLWVHLRPADTVTTVFLQISR IKATTEGNSR IRILSLKIDVASGASSWQSVDINQLLKTWLRQPET

O. tshawytscha I LHAQLWVHLRPADTVTTVFLQ ISR IKATTEGNSR IRILSLKIDVASGASSWQSVDINQLLKTWLRQPET o. clarki I LHAQLWVHLRPADTVTTVFLQ ISR I KATTEGNSR IRILSLKIDVASGASSWQSVDINQLLKTWLRQPET

O. kisutch I HAQLWVHLRPADTVTTVFLQ ISR IKATTEGNSRIRILSLKIDVVSGSSSWQSVDN-QLLKTWLHQPET

O. nerka ILHAOLWVHLRPADTVTTVFLOISR IKATTEGNSRIRILSLKIDVASGASSWOSVDINOLLKTWLROPET

S. salar I LHAQLWVHLRPADTVTTVFLQISR IKATTEGNSRIRILSLKIDVASGASSWQSVDINQLLKTWLRQPET

Sv. tontinalis I LHAQLWVHLRPADTVTTVFLQISR IKATTEGNSR IR I LSLKIDVASGASSWQSVDINQLLKTWLRQPET

c. lavaretus I L HAQ LWVHLRPADTVTTVFLQISR I KATTEGNSGIRILSLKIDVASGASSWQSVDINQLLKTWLRQPET

c. clupeatormis I LHAQLWVHLRPADTVTTVFLQMSR I KATTEGNSR IRILSLKIDVASGASSWQSVDINQLLKTWLRQPET

T. arcticus I LHAQLWVHLRPADTVTTVFLQ ISR I KATTEGNSR IR I LSLK INVASGASSWQNVDINQLLKAWLRQPET

T. thylamus I LHAQLWVHLRPADTVTTVFLQISRIKATTEGNSRIRILSLKINVASGASSWQNVDINQLLKAWLRQPET

P. williamsoni I LHA LWVHLRPADTDTTVFLQ ISR I KATTEGNSR IRILSLKIDVASGASSWQSVDINQLLKTWLRQPET

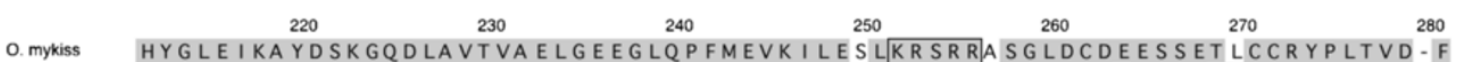

O. tshawyscha HYGLEIKAYDSKGQDLAVTVAELGEEGLQPFMEVKILESLKRSRRASGLDCDQESSESRCCRYPLTVD-F

0. clarki HYGLEIKAYDSKGQDLAVTVAELGEEGLQPFMEVK I LESLIKRSRRASGLDCDEESSETLCCRYPLPVD-F

O. kisutch HYGLEIKAYDSKRQDLAVTVAELGEEGLQLFMEVK ISENLKRSRRDSALDCDEESSETLCCRYPLTID-F

O. nerka HYGLEIKAYDSKGQDLAVTVAELGEEGLQPFMEVKILESLKRSRRASGLDCDEESSESRCCRYPLTVD-F

S. salar HYGLEIKAYDSKGQDLAVTVAELGEEGLQPFVEVKILDNLKRSRRASGLDCDEESSETRCCRYPLTVD-F

Sv. tontinalis HYGLEIKAYDSKGQDLAVTVAELGEEGLQPFVEVKILENLKRSRRASGLDCDEESSETRCCRYPLTVD-F

C. lavaretus HYGLEIKAYDSKGQDLAVTVAELGEEGLQPFMEVKILENLKRSRRDSGLDCDEESSETRCCRYPLTVD-F

c. clupeatormis HYGLE IKA YDSKGQDLAVTVAELGEEGLQPFMEVK I LENLKRSRRDSGLDCDEESSETRCCRYPLTVD-F

T. arcticus HYGLEIKAYDSNGKDLAVTVVELGEEGLQPFMEVKILENLRRSRRDSGLDCDEESSETRCCRYPLTVDWL

T. thylamus HYGLEIKAYDSNGKDLAVTVVELGEEGLQPFMEVK I LENLRRSRRDSGLDCDEESSETRCCRYPLTVDWL

P. williamsoni HYGLEIKAYDSKGQDLAVTVAELGEEGLQPFMEVK I LENLKRSRR DSGLDCDEESSETRCCRYPLTVD-F

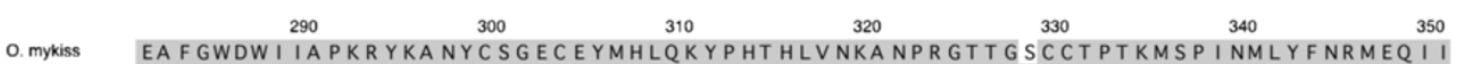

O. tshawyscha EAFGWDWI IAPKRYKANYCSGECEYMHLQKYPHTHLVNKANPRGTTGPCCTPTKMSPINMLYFNRMEOI I

0. clarki EAFGWDWI I A PKRYKANYCSGECEYMHLQKYPHTHLVNKANPRG ITGPCCTPTKMSP INMLYFNRMEQ I I

O. kisutch EAFGWDWIIAPKRFKANYCSGECEYMHLQKYPHTHLVNKA SAGVPQGPCCTP.

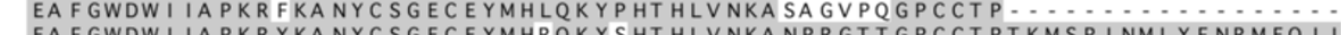

S. salar EAFGWDWI IAPKRYKANYCSGECEYMHLQKYPHTHLVNKANPRGTTGPCCTPTKMSPINMLYFNRMEQI I

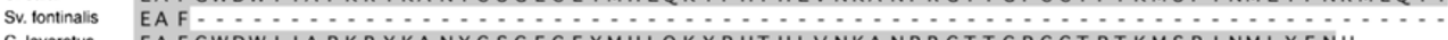

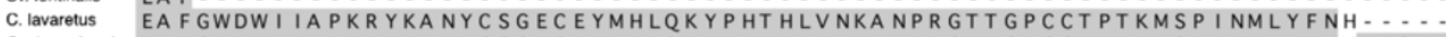

C. clupeatormis EA F GWDW I IAPKR YKA NYCSGECEYMHLQKYPHTHLVNKANPRGTTGPCCTPTKMSP INMLYFNHMEQI I

T. arcticus EAFGWDWIIAPKRYKANYCSGECEYMHLQKYPHTHLVNKANPQGTTGPCCTPTKMSP I NMLYFNHMEQ I I

T. thylamus EAFGWDW I I APKRYKANYCSGECEYMHLQKYPHTHLVNKANPQGTTGPCCTPTKMSP INMLYFNHMEQ I I

P. williamsoni EA F GWDW I I A PKRYKANYCSGECEYMHLQKYPHTHLVNKANPRGATGPCCTPTKMSPINMHYFNRMEQ I I

\begin{tabular}{|c|c|}
\hline & 360 \\
\hline O. mykiss & YGK IPSMVVDHCGCS \\
\hline O. tshawytscha & YGK IPSMVVDHCGCS \\
\hline O. clarki & YGKIPSMVVDHCGCS \\
\hline O. kisutch & $\cdots \cdots$ \\
\hline O. nerka & YGK I PSMVVDHCGCS \\
\hline S. salar & YGK IPSMVVDHCGCS \\
\hline Sv. fontinalis & $\ldots \ldots$ \\
\hline C. lavaretus & $\ldots \ldots \ldots$ \\
\hline C. clupeatormis & YGK I PSMVVDRCGCS \\
\hline T. arcticus & YGK IPSMVVDHCGCS \\
\hline T. thylamus & YGK IPSMVVDHCGCS \\
\hline P. williamsoni & YGKIPSMVVDHCGCS \\
\hline
\end{tabular}

Figure 4 (See legend on next page.) 
(See figure on previous page.)

Figure 4 Multiple sequence alignment of salmonid MSTN-2a paralogs. Amino acid positions are numbered above the sequence line, taxa are indicated to the left and amino acid identities are shaded gray. Gaps and unsequenced regions are indicated by dashes and the furin/PC cleavage site is boxed.

Evidence for variable rate changes (and corresponding selection) along different branches was detected in the MSTN-1a/1b and MSTN-2a/2b trees by measuring $\mathrm{dN} /$ dS ratios using both a branch and a branch-site analysis.
In the branch-site analysis (Tables 2, 3), all branches displaying a statistically significant $\mathrm{dN} / \mathrm{dS}$ value greater than 1 are locations with substantial increases in rate variation and are possible sites for relaxed selective

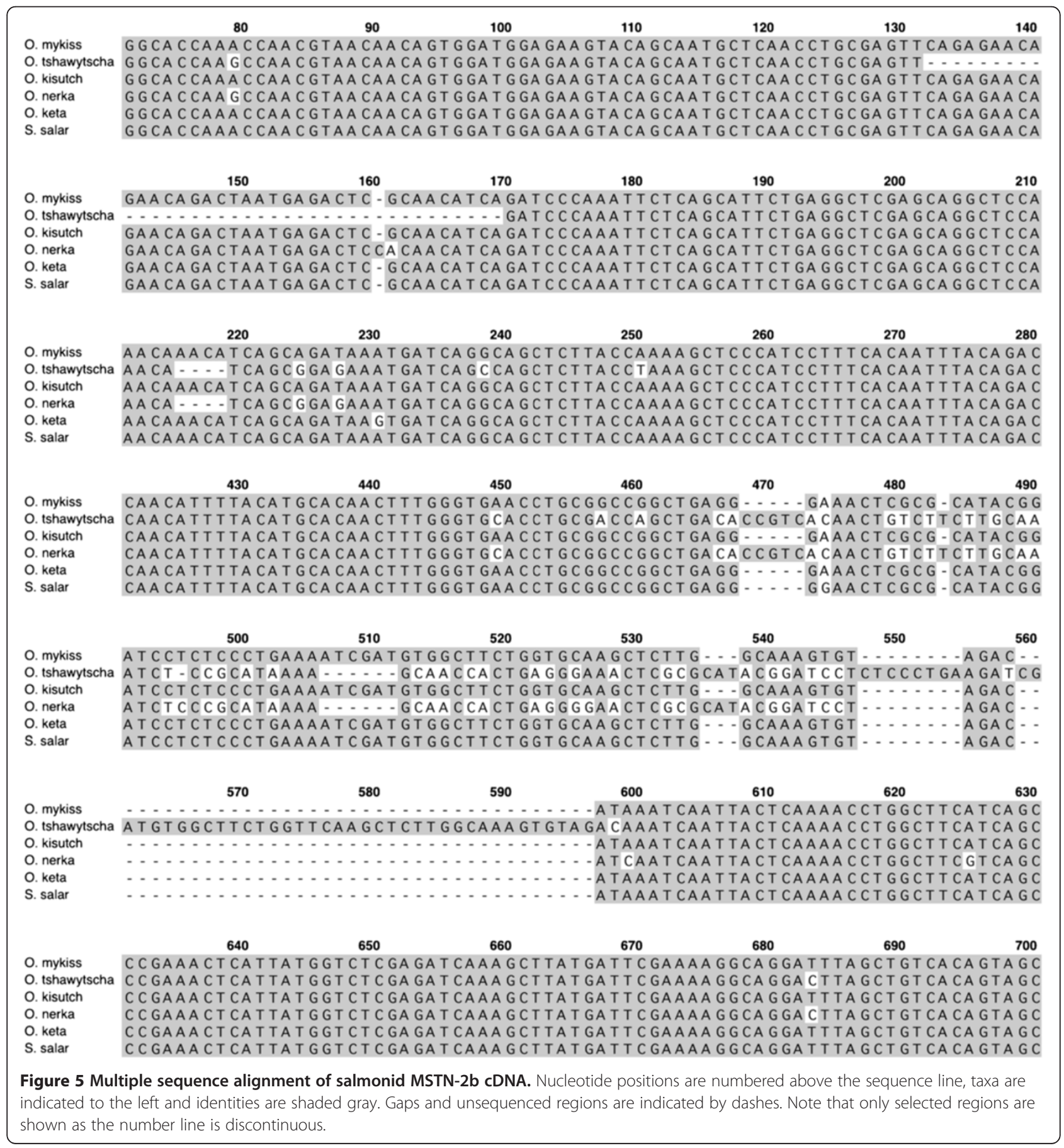




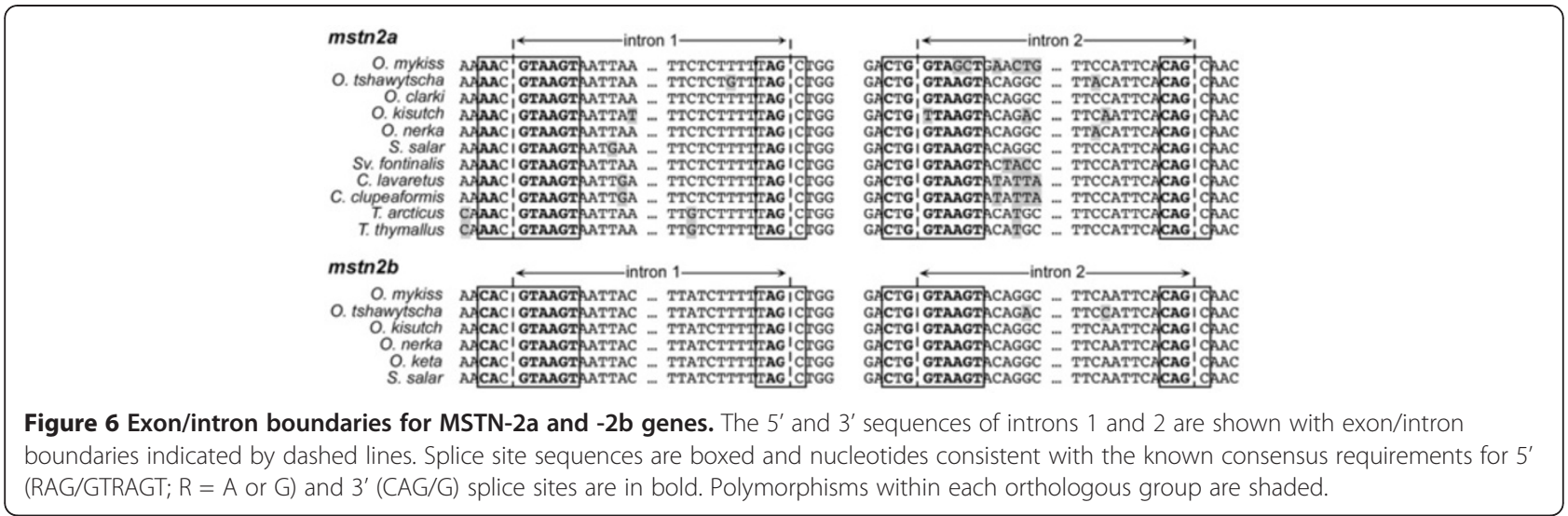

constraint or positive selection. Within the MSTN-1a/1b tree, two branches showed evidence of variable rate change, most notably separating the mstn $1 a$ genes from the $m s \operatorname{tn} 1 b$ genes. The MSTN-2a/2b tree also showed signals of relaxed selective constraint, with four branches demonstrating a statistically significant $\mathrm{dN} / \mathrm{dS}$ value greater than 1. Interestingly, one of the four branches showing a signal for $\mathrm{dN} / \mathrm{dS}$ was the branch leading to $O$. kisutch mstn $2 a$, which was placed outside of all of the other mstn $2 a$ genes, further strengthening the conclusion that this gene has evolved differently than other $m s t n 2 a$ genes. The Bayes Empirical Bayes (BEB) analysis revealed one site within the MSTN-1a/1b tree under positive selection with a probability greater than 95 (Additional file 4). This site, position 185 on branch 34 is located within the propeptide (positions 24-266) of the protein. The MSTN-2a/2b analysis revealed several sites under positive selection all located within the propeptide (Additional file 4) located on branches 7, 18, and 19. None of the sites (listed in Additional file 4) determined to be under positive selection correlated with the sites found by Tellgren, et al., 2004 for orthologous divergence in ruminant Artiodactyls. Within the branch model of MSTN-2a/2b (Figure 9, dN/dS values calculated across each branch in Figure 8), eleven branches had values greater than 1, although the test does not allow for establishing individual branches as being significantly greater than 1 . Most notable among the branches is a high signal for rate variation within the $m s t n 2 b$ phylogeny, presumably all pseudogenes, which is indicative of relaxed selection. No figure is present for the branch model of the MSTN-1a/1b tree as there was no statistical support for a free-ratios model over the single-ratio model. SplitsTree was run to test if there was strong support for a single phylogenetic tree that could explain the underlying sequence data in mstn1

Table 1 Putative branch points in salmonid mstn2 introns

\begin{tabular}{|c|c|c|c|c|}
\hline species & mstn2a Intron 1 & mstn $2 a$ Intron 2 & $m s t n 2 b$ Intron 1 & $m s t n 2 b$ Intron 2 \\
\hline \multirow[t]{2}{*}{ O. mykiss } & NOT FOUND & GGCTGAC 919 (50) & TTCTAAC 484 (138) & NOT FOUND \\
\hline & & TACTAAT 955 (86) & & \\
\hline \multirow[t]{2}{*}{ O. tshawytscha } & AACTGAC 457 (117) & GGCTGAC 982 (23) & TTCTAAC 443 (138) & NOT FOUND \\
\hline & TTCTAAC 478 (138) & & & \\
\hline O. clarki & TTCTAAC 478 (138) & GGCTGAC 891 (23) & & \\
\hline O. kisutch & TTCTAAC 479 (139) & NOT FOUND & TTCTAAC 484 (138) & NOT FOUND \\
\hline O. keta & & & TTCTAAC 484 (138) & NOT FOUND \\
\hline O. nerka & TTCTAAC 478 (138) & GGCTGAC 892 (23) & TTCTAAC 433 (138) & NOT FOUND \\
\hline S. salar & TTCTAAC 481 (138) & GGCTGAC 895 (23) & TTCTAAC 484 (138) & NOT FOUND \\
\hline Sv. fontinalis & TTCTAAC 361 (138) & NOT FOUND & & \\
\hline C. lavaretus & TCCTAAC 463 (123) & NOT FOUND & & \\
\hline C. clupeaformis & TTCTAAC 463 (123) & NOT FOUND & & \\
\hline T. arcticus & TTCTAAC 424 (81) & NOT FOUND & & \\
\hline T. thymallus & TTCTAAC 424 (81) & NOT FOUND & & \\
\hline
\end{tabular}

Polymorphysms in the known consensus (TNCTRAY; $\mathrm{N}=$ any nucleotide, $\mathrm{R}=\mathrm{A}$ or $\mathrm{G}, \mathrm{Y}=\mathrm{C}$ or $\mathrm{T}$ ) are bold. Numbers represent the distance (bp) of the first nucleotide within putative sites from the initiator or the $5^{\prime}$ intron boundary (parenthesis). In some instances, two putative branch points were identified. 


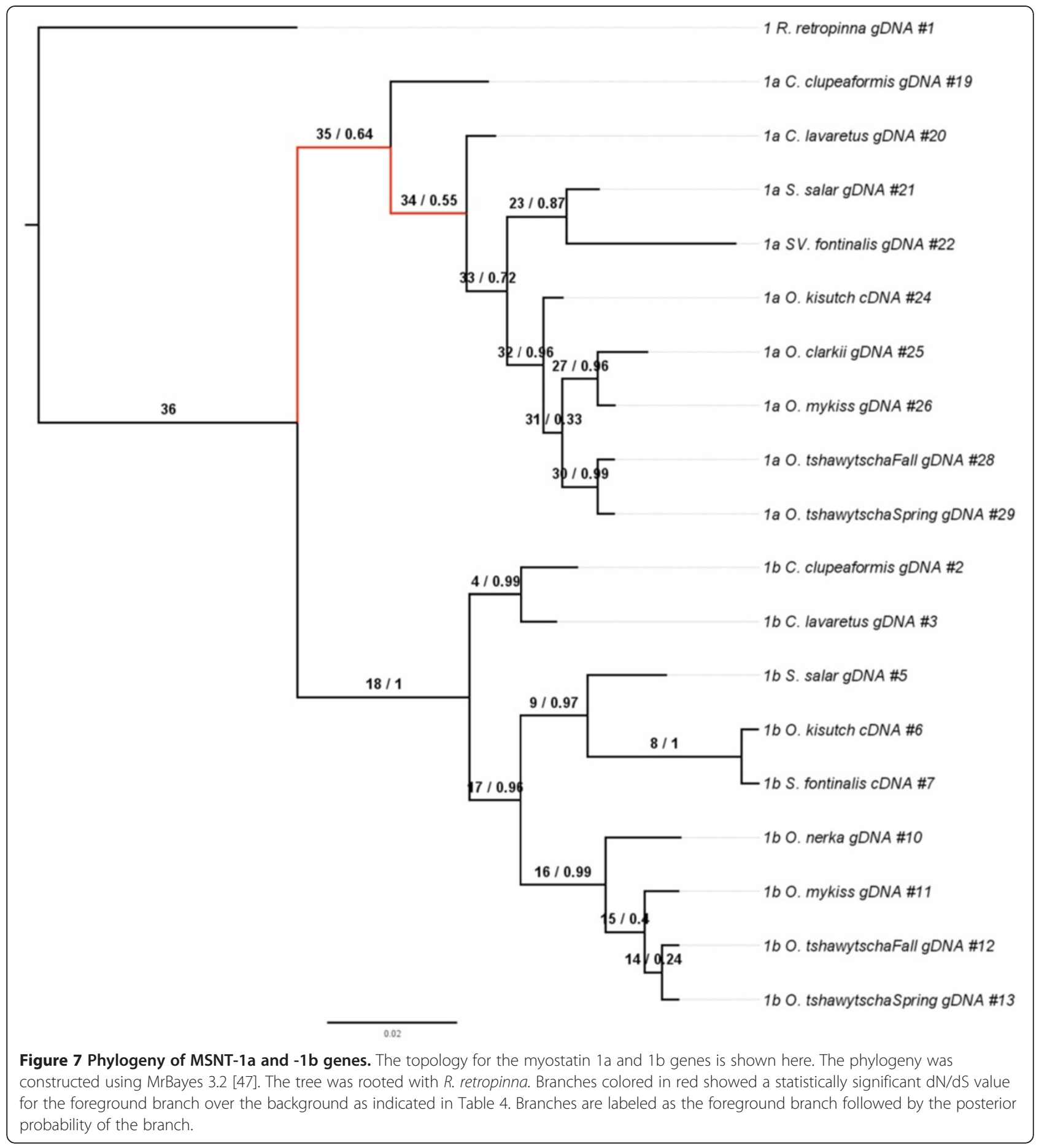

and mstn2 (Figures $10 \& 11$ ). This analysis revealed highly non-tree like structures for both paralog groups and is suggestive of hybridization, incomplete lineage sorting or stochastic phylogenetic incongruence.

\section{Discussion}

Gene duplication is an important process that alters gene function often via changes in gene structure/ sequence, patterns of gene expression and as recently determined, altered transcript processing [19-21]. Examples of such differences are seen in the salmonid myostatin gene family, suggesting that it is diverging via a combination of neofunctionalization and subfunctionalization. The former involves the introduction of new functions for duplicate genes whereas with subfunctionalization, duplicate genes specialize to form 


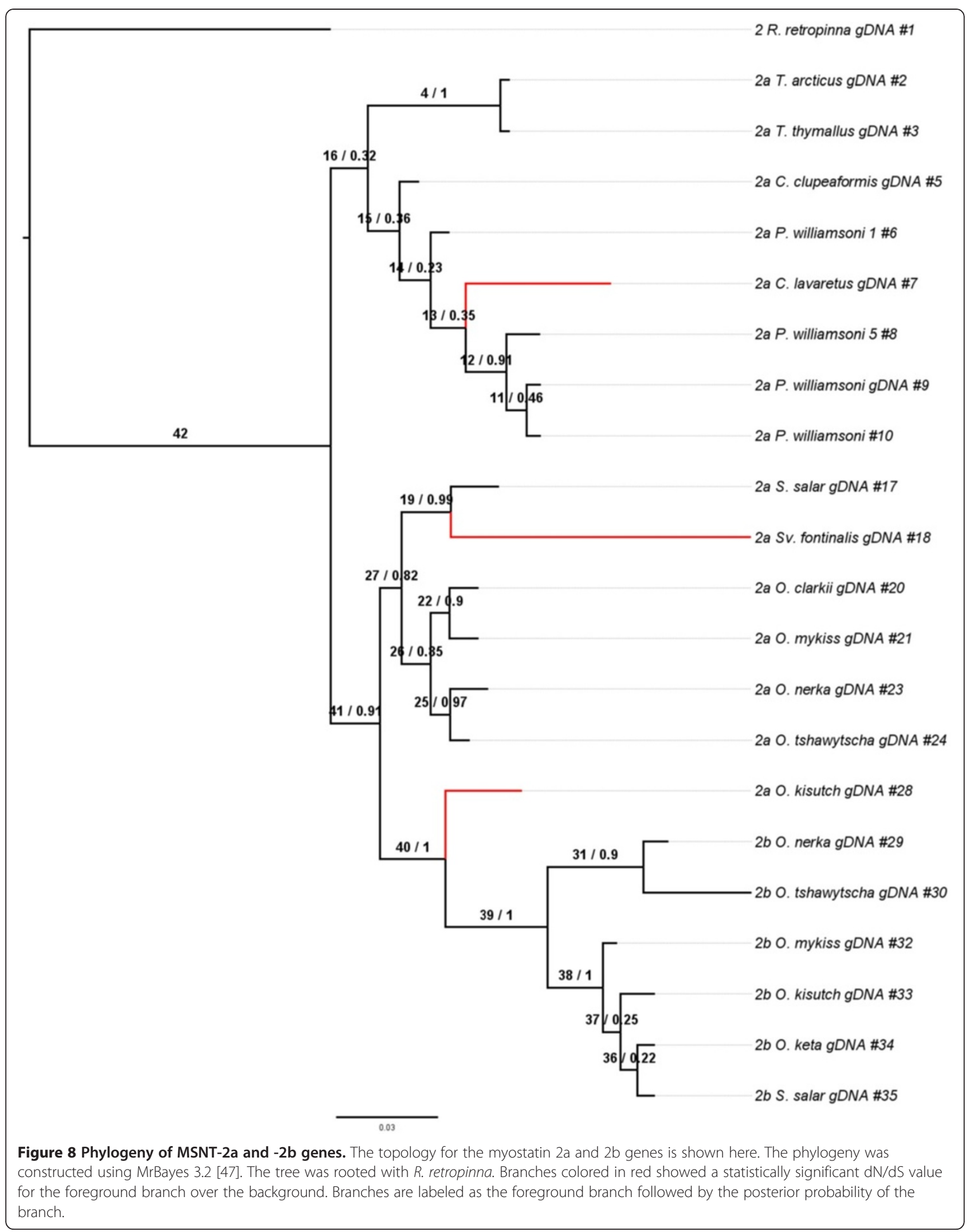


Table 2 Likelihood ratio test analysis and dN/dS ratios for MSTN-1a and MSTN-1b

\begin{tabular}{|c|c|c|c|c|c|c|}
\hline Foregroun branch & P-value & Likehood ratio test & $\mathrm{p}_{0}$ & $\mathrm{p}_{1}$ & $w_{0}$ & $W_{2}$ \\
\hline 1 & 1 & 0 & 0.78604 & 0.0735 & 0.06418 & 1 \\
\hline 2 & 1 & 0 & 0.91449 & 0.08551 & 0.06418 & 1 \\
\hline 3 & 1 & 0 & 0.87254 & 0.08151 & 0.06349 & 1 \\
\hline 4 & 1 & 0 & 0.84418 & 0.0783 & 0.06256 & 1 \\
\hline 5 & 1 & 0 & 0.75124 & 0.07563 & 0.05589 & 1 \\
\hline 6 & 1 & 0 & 0.7823 & 0.07315 & 0.06418 & 1.0937 \\
\hline 7 & 1 & 0 & 0.78494 & 0.0734 & 0.06418 & 1.0519 \\
\hline 8 & 0.837932705 & 0.041836 & 0.8317 & 0.06936 & 0.06127 & 1.53474 \\
\hline 9 & 0.435190969 & 0.60893 & 0 & 0 & 0.05808 & 116.56426 \\
\hline 10 & 1 & 0 & 0.91449 & 0.08551 & 0.06418 & 1 \\
\hline 11 & 0.480047236 & 0.498756 & 0 & 0 & 0.05991 & 154.56582 \\
\hline 12 & 0.998871621 & $2.00 \mathrm{E}-06$ & 0.78682 & 0.07357 & 0.06418 & 1.05741 \\
\hline 13 & 0.998871621 & $2.00 \mathrm{E}-06$ & 0.78321 & 0.07324 & 0.06418 & 1 \\
\hline 14 & 1 & 0 & 0.91449 & 0.08551 & 0.06418 & 1 \\
\hline 15 & 1 & 0 & 0.91449 & 0.08551 & 0.06418 & 1 \\
\hline$\overline{16}$ & 1 & 0 & 0.91449 & 0.08551 & 0.06418 & 1 \\
\hline 17 & 1 & 0 & 0.91449 & 0.08551 & 0.06418 & 1 \\
\hline 18 & 0.02608246 & 4.950544 & 0.91976 & 0.07657 & 0.06708 & 998.99999 \\
\hline 19 & 1 & 0 & 0.77391 & 0.07197 & 0.06306 & 1 \\
\hline 20 & 1 & 0 & 0.91449 & 0.08551 & 0.06418 & 1 \\
\hline 21 & 0.80351773 & 0.0619 & 0 & 0 & 0.06339 & 104.27124 \\
\hline 22 & 1 & 0 & 0.91449 & 0.18551 & 0.06418 & 1 \\
\hline 23 & 1 & 0 & 0.7271 & 0.06908 & 0.05868 & 1 \\
\hline 24 & 1 & 0 & 0.91149 & 0.08551 & 0.06418 & 1 \\
\hline 25 & 1 & 0 & 0.91149 & 0.08551 & 0.06418 & 1 \\
\hline 26 & 1 & 0 & 0.91149 & 0.08551 & 0.06418 & 1 \\
\hline 27 & 1 & 0 & 0.91149 & 0.08551 & 0.06418 & 1 \\
\hline 28 & 0.998871621 & $2.00 \mathrm{E}-06$ & 0.78417 & 0.07332 & 0.16418 & 1.04859 \\
\hline 29 & 0.998404232 & 4.00E-06 & 0.78127 & 0.07305 & 0.16418 & 1.06811 \\
\hline 30 & 1 & 0 & 0.91449 & 0.08551 & 0.06418 & 1 \\
\hline 31 & 1 & 0 & 0.77679 & 0.07263 & 0.16418 & 1.34502 \\
\hline 32 & 1 & 0 & 0.91149 & 0.08551 & 0.16418 & 1 \\
\hline 33 & 1 & 0 & 0.91149 & 0.8551 & 0.06418 & 1 \\
\hline 34 & 0.001154703 & 10.561454 & 0.9179 & 0.72176 & 0.06509 & 103.9286 \\
\hline 35 & 0.009387076 & 10.945966 & 0.91398 & 0.07251 & 0.0627 & 999 \\
\hline 36 & 1 & 0 & 0.78794 & 0.07368 & 0.06418 & 1 \\
\hline
\end{tabular}

Summary statistics generated for each foreground branch using a Branch-site test. Branches in bold and italics are are statistically significant with a dN/dS ratio greater than 1. Branch numbers are labeled in Figure 7.

complementary functions [17]. For example, the expanded repertoire of expression patterns in the composite of fish myostatin genes relative to mammalian homologs suggests that expression profiles have neofunctionalized following the teleost genome duplication [22]. How this interplays with the second whole genome duplication in salmonids [23] is unclear.

Generally, the evolutionary dynamics of duplicate genes resulting from whole genome duplications differ from those that occur after small-scale events [24,25] and result in a higher probability of long-term retention. In fact, duplicate genes are typically retained over long evolutionary periods only after some divergence of function, which as discussed, can result from changes in gene structure, promoter activity and/or transcript processing. Gene expression profiles and subfunctionalization tend to evolve faster than coding sequence functions and neofunctionalization due to the greater potential for deleterious versus advantageous changes [26-28]. Of course, the vast majority of duplicate genes is not retained, but 
Table 3 Likelihood ratio test analysis and dN/dS ratios for MSTN-2a and MSTN-2b

\begin{tabular}{|c|c|c|c|c|c|c|}
\hline Foreground branch & P-value & Likehood ratio test & $\mathrm{p}_{0}$ & $p_{1}$ & $w_{0}$ & $W_{1}$ \\
\hline 1 & 0.992953366 & $7.80 \mathrm{E}-05$ & 0.85159 & 0.14839 & 0.11865 & 1 \\
\hline 2 & 0.9953476 & $3.40 \mathrm{E}-05$ & 0.85149 & 0.14837 & 0.11865 & 1 \\
\hline 3 & 0.9953476 & $3.40 \mathrm{E}-05$ & 0.85148 & 0.14837 & 0.11865 & 1 \\
\hline 4 & 1 & 0 & 0.60715 & 0.10719 & 0.10494 & 1 \\
\hline 5 & 1 & 0 & 0.70568 & 0.12296 & 0.11865 & 1.04992 \\
\hline 6 & 0.998871621 & $2.00 \mathrm{E}-06$ & 0.7009 & 0.12213 & 0.11865 & 1.04909 \\
\hline 7 & $3.42 \mathrm{E}-11$ & 43.922434 & 0.8387 & 0.12582 & 0.11621 & 518.94859 \\
\hline 8 & 1 & 0 & 0.85161 & 0.14839 & 0.11865 & 1 \\
\hline 9 & 1 & 0 & 0.85161 & 0.14839 & 0.11865 & 1 \\
\hline 10 & 0.993717516 & $6.20 \mathrm{E}-05$ & 0.8516 & 0.14839 & 0.11865 & 1 \\
\hline 11 & 1 & 0 & 0.85161 & 0.14839 & 0.11865 & 1 \\
\hline 12 & 1 & 0 & 0.69073 & 0.12412 & 0.11458 & 1 \\
\hline 13 & 1 & 0 & 0.6942 & 0.12096 & 0.11862 & 1.20916 \\
\hline 14 & 1 & 0 & 0.70098 & 0.1214 & 0.11862 & 1.05347 \\
\hline 15 & 1 & 0 & 0.85161 & 0.14839 & 0.11865 & 1 \\
\hline 16 & 0.625942013 & 0.237604 & 0.70371 & 0.12262 & 0.11865 & 1.12316 \\
\hline 17 & 1 & 0 & 0.30086 & 0.05619 & 0.10976 & 1 \\
\hline 18 & $1.70 E-12$ & 49.80162 & 0.83059 & 0.09738 & 0.11719 & 146.51077 \\
\hline 19 & 0.010178788 & 6.603336 & 0.83919 & 0.14111 & 0.11848 & 999 \\
\hline 20 & 1 & 0 & 0.85161 & 0.14839 & 0.11865 & 1 \\
\hline 21 & 1 & 0 & 0.53537 & 0.09578 & 0.11454 & 1 \\
\hline 22 & 1 & 0 & 0.85161 & 0.14839 & 0.11865 & 1 \\
\hline 23 & 0.6663122 & 0.185568 & 0.77072 & 0.13927 & 0.11378 & 5.51208 \\
\hline 24 & 1 & 0 & 0.727 & 0.12965 & 0.11646 & 1 \\
\hline 25 & 1 & 0 & 0.85161 & 0.14839 & 0.11865 & 1 \\
\hline 26 & 1 & 0 & 0.85161 & 0.14839 & 0.11865 & 1 \\
\hline 27 & 1 & 0 & 0.85161 & 0.14839 & 0.11865 & 1 \\
\hline 28 & 4.37E-05 & 16.704412 & 0.83602 & 0.15333 & 0.1199 & 999 \\
\hline 29 & 1 & 0 & 0.85161 & 0.14839 & 0.11865 & 1 \\
\hline 30 & 1 & 0 & 0.85161 & 0.14839 & 0.11865 & 1 \\
\hline 31 & 1 & 0 & 0.85161 & 0.14839 & 0.11865 & 1 \\
\hline 32 & 1 & 0 & 0.70563 & 0.12296 & 0.11865 & 1.08115 \\
\hline 33 & 0.460406603 & 0.544906 & 0 & 0 & 0.11556 & 140.21885 \\
\hline 34 & 0.994358151 & $5.00 \mathrm{E}-05$ & 0.70444 & 0.12275 & 0.11865 & 1.06825 \\
\hline 35 & 1 & 0 & 0.85161 & 0.14839 & 0.11865 & 1 \\
\hline 36 & 1 & 0 & 0.70412 & 0.12269 & 0.11865 & 1.05 \\
\hline 37 & 1 & 0 & 0.70542 & 0.12292 & 0.11865 & 1.05032 \\
\hline 38 & 1 & 0 & 0.85161 & 0.14839 & 0.11865 & 1 \\
\hline 39 & 1 & 0 & 0.69477 & 0.12223 & 0.11202 & 1 \\
\hline 40 & 1 & 0 & 0 & 0 & 0.10345 & 1 \\
\hline 41 & 1 & 0 & 0.74051 & 0.12707 & 0.11695 & 1 \\
\hline 42 & 1 & 0 & 0.73126 & 0.12428 & 0.11865 & 1 \\
\hline
\end{tabular}

Summary statistics generated for each foreground branch using a Branch-site test. Branches in bold and italics are statistically significant with a dN/dS ratio greater than 1. Compare foreground branches with Figures 8,9 . 


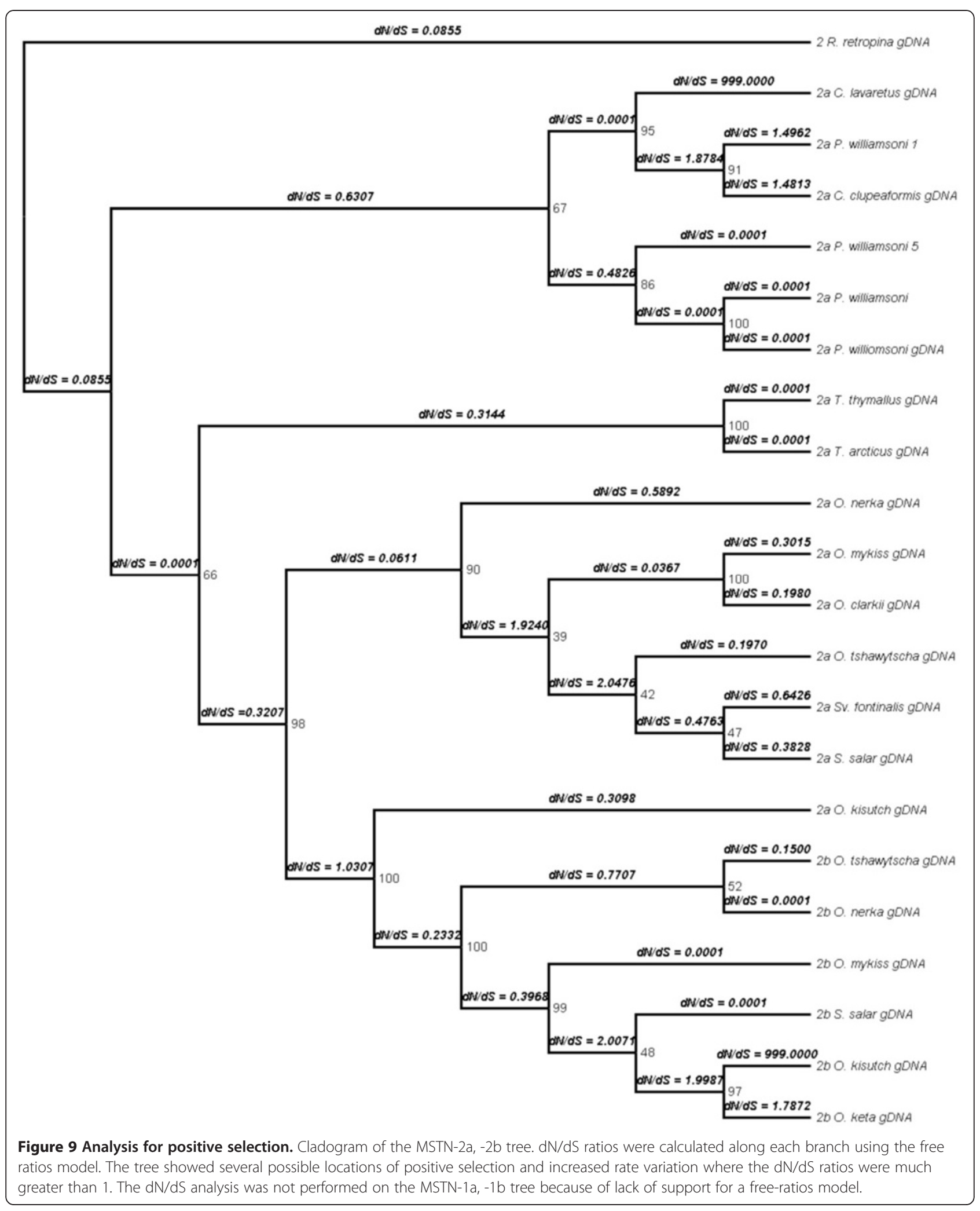




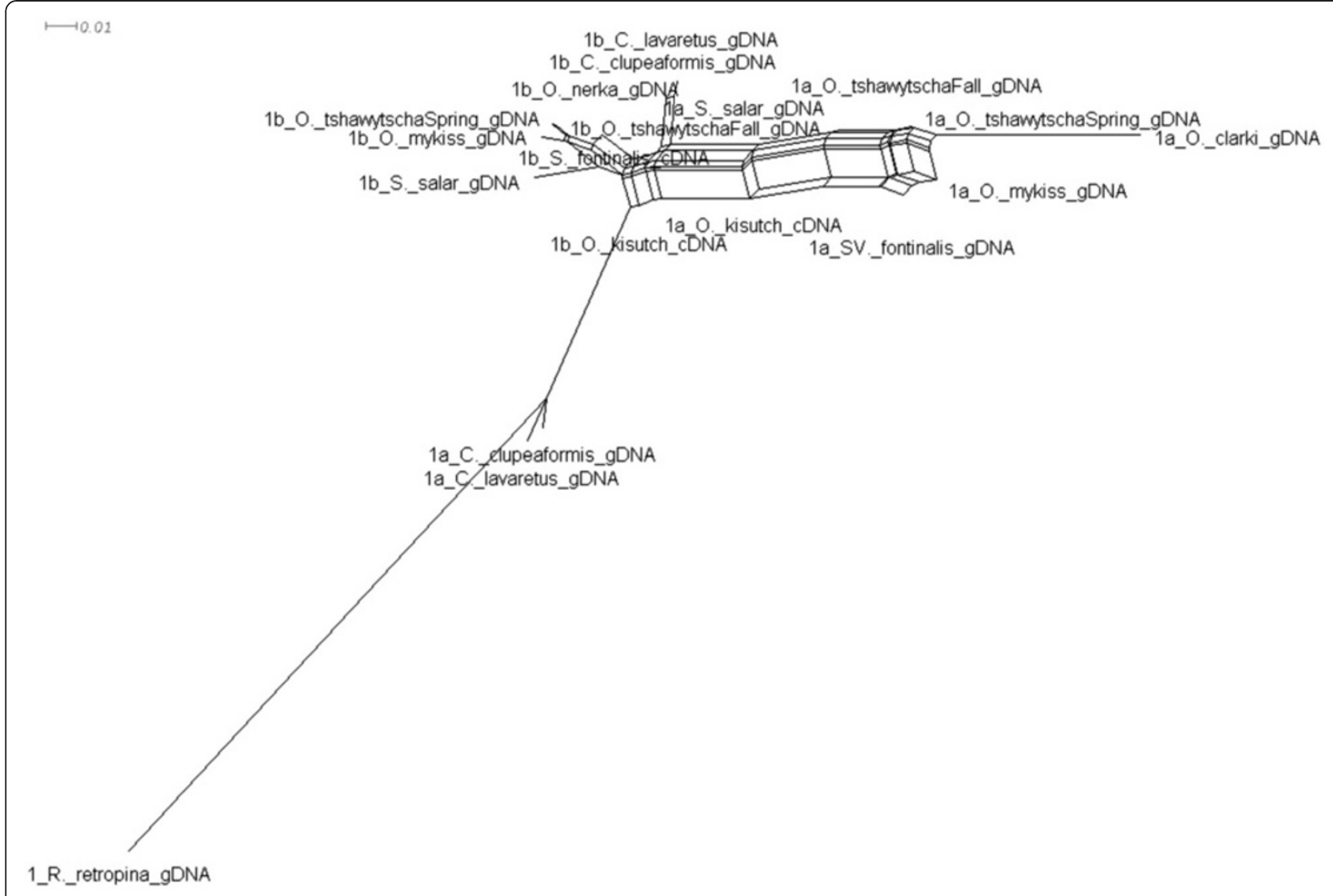

Figure 10 Splitstree for MSTN-1a, -1b. Splitstree built using SplitsTree4. The tree represents the possible amount of hybridization that has occurred within MSTN-1a, $-1 b$ in salmonids.

is rapidly nonfunctionalized, as with $m s t n 2 b$. Most studies investigating the underlying mechanisms of nonfunctionalization have focused primarily on coding sequence mutations. Thus, the fact that changes in transcript processing contributed to the nonfunctionalization of both mstn2 paralogs is truly novel especially as it appears to predate the coding sequence changes and results in the tissue-specific nonfunctionalization of mstn2a. In fact, alternative processing has only recently been demonstrated to influence gene fate [29-31], which further illustrates the importance of studying the salmonid myostatin gene family. Future studies are nevertheless needed to confirm that the mstn $2 a$ and $m s t n 2 b$ transcript processing patterns are indeed conserved among all salmonids. Nevertheless, the shared lack of intact motifs necessary for removing intronic sequences, in mstn 2 paralogs cloned from all three subfamilies, suggests that the patterns are indeed conserved among all salmonids.

We recently demonstrated subfunctionalization of rainbow trout mstn $1 a$ and $m s t n 2 a$ specifically in their ability to regulate the differentiation of primary myosatellite cells [11]. In mammals, myostatin is upregulated in differentiating muscle cells and by insulin-like growth factor (IGF)-I [32-35], a known endocrine regulator of myogenesis [36]. It is therefore believed to partly mediate the actions of IGF-I on muscle cell differentiation [1,37-39]. This represents an ancestral state where a single myostatin gene serves multiple functions. The process is more complicated in rainbow trout as mstnla appears to stimulate differentiation in response to serum whereas $m s t n 2 a$ in response to IGF-I. The combined actions of a single myostatin gene in mammals have therefore subfunctionalized in rainbow trout and possibly other salmonids with regard to this function. The relative changes in mstn $1 b$ expression for the most part mirror those of mstn1a, although $m s t n 1 a$ levels always exceed those of $m s \operatorname{tn} 1 b$. This is suggestive of functional redundancy, at least in regards to muscle cell differentiation.

Neofunctionalization and subfunctionalization in the expression of the different paralogs is due to differences in promoter structure/function as the genes are differentially expressed by these different myogenic conditions. Alternative processing of $m s t n 2 a$ transcripts plays a role as well as it is also stimulated by IGF-I. Nova proteins are known regulators of alternative processing in the 


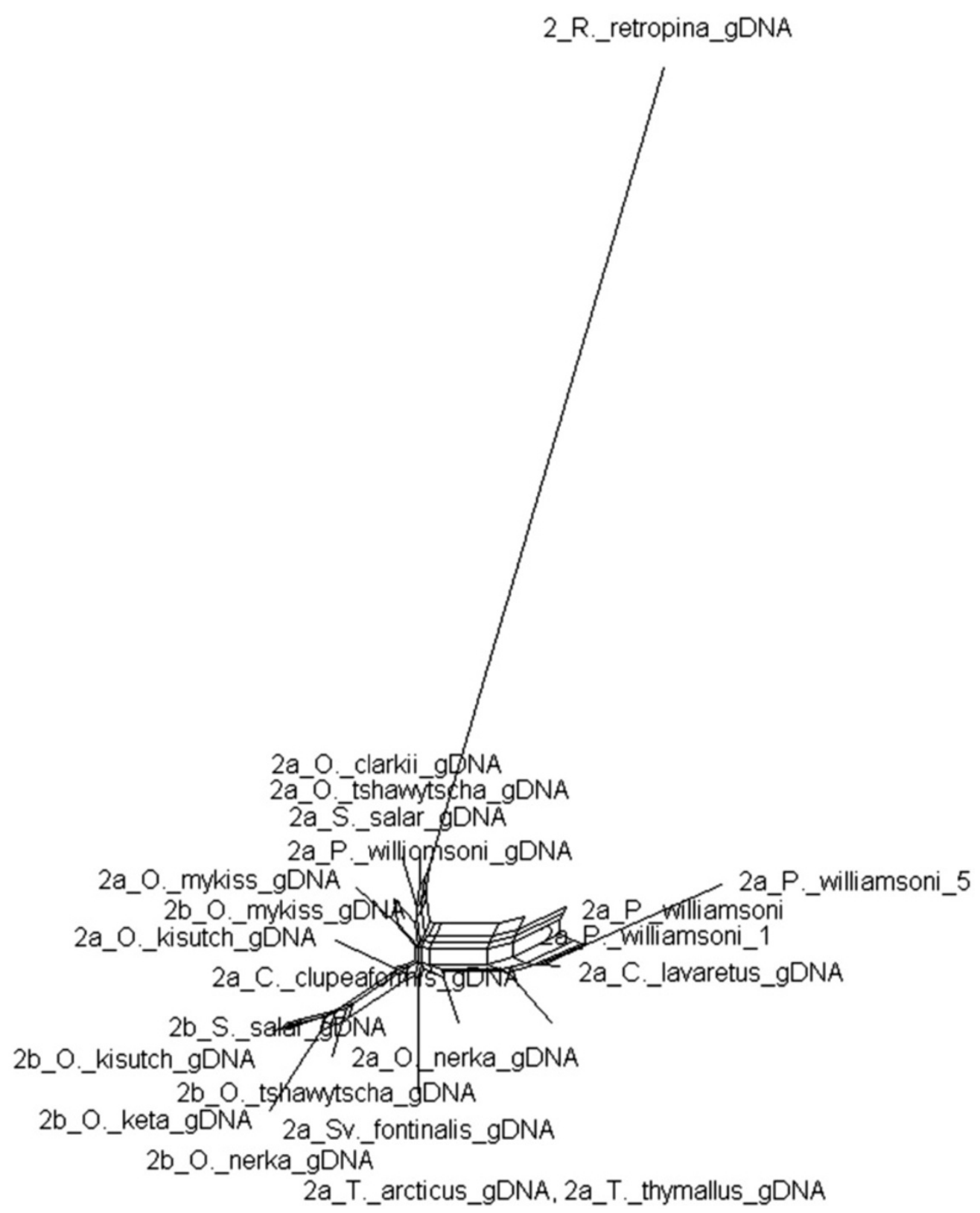

Figure 11 Splitstree for MSTN-2a, -2b. Splitstree built using SplitsTree4. The tree represents the possible amount of hybridization that has occurred within MSTN-2a, $-2 b$ in salmonids.

brain, the only tissue where mstn $2 a$ transcripts are fully processed without IGF stimulation, and recognize YCAY motifs that can either direct or misdirect the splicesome [40-42]. A survey of such motifs throughout the mstn $2 a$ and $m s \sin 2 b$ genes identified several putative binding sites that are unique to $m s t n 2 a$, particularly in the second intron (Table 4). It is unknown whether Nova proteins truly regulate mstn $2 a$ processing in brain, muscle or in response to IGF-I. The suggested model of neofunctionalization or subfunctionalization via alternative processing, however, is extremely novel and the possible contributions of Nova proteins is at least plausible and best of all, testable. It is also amenable to computational approaches that could track selection events that influence transcript processing across taxa and thus, the molecular mechanisms of functional change itself.

Analyses of the mstn1a, mstn1b, and mstn $2 a$ phylogenetic relationships were generally consistent with the established salmonid relationships. The only exception was for O. kisutch which, based on the topologies of these coding genes, diverged before the other Oncorhynchus species examined (Figures $7 \& 8$, Additional 
Table 4 Number and location of YCAY motifs in salmonid mstn2 genes

\begin{tabular}{|c|c|c|c|c|c|c|c|c|c|c|c|c|}
\hline & O myk & O ner & O tsh & O kis & S sal & O ket & O cla & Sv fon & $T$ thy & $T$ arc & C clu & Clav \\
\hline Ex 1 & $8 / 8$ & 8/NA & $8 / 7$ & $8 / 8$ & $8 / 8$ & NA/8 & 8 & NA & 9 & 9 & 8 & 8 \\
\hline $\ln 1$ & $4 / 4$ & $4 / 4$ & $4 / 3$ & $4 / 4$ & $4 / 4$ & $\mathrm{NA} / 4$ & 4 & 4 & 1 & 1 & 3 & 3 \\
\hline Ex 2 & $6 / 5$ & $7 / 7$ & $7 / 7$ & $7 / 5$ & $7 / 5$ & NA/5 & 6 & 6 & 6 & 6 & 6 & 6 \\
\hline $\ln 2$ & $13 / 7$ & $13 / 8$ & $12 / 6$ & $7 / 6$ & $13 / 8$ & NA/6 & 13 & 13 & 11 & 11 & 8 & 8 \\
\hline Ex 3 & $10 / 10$ & 10/NA & $10 / 8$ & $7 / 4$ & $9 / 9$ & NA/5 & 9 & NA & 12 & 11 & 10 & NA \\
\hline Total & $41 / 34$ & 42/NA & $41 / 31$ & $33 / 27$ & $41 / 34$ & NA/28 & 40 & N/A & 39 & 38 & 35 & N/A \\
\hline
\end{tabular}

Numbers are for mstn2a/mstn2b, respectively. Single numbers/cell represent mstn2a only. (Y, $\mathrm{C}$ or T; NA, not available due to incomplete sequences).

Abbreviations: O, Oncorhynchus; myk, mykiss; ner, nerka; tsh, tshawytscha; kis, kisutch; S sal, Salmo salar; ket, keta; cla, clarki; Sv fon, Salvelinus fontinalis; T, Thymallus; thy, thymallus; arc, arcticus; C, Coregonus; clu, clupeaformis; lav, lavaretus.

files $1 \& 2$ ). This was not supported by the topologies of the pseudogene $m s t n 2 b$, which may be more meaningful. Indeed, pseudogenes are by definition non-functional and are not phenotypically expressed. Unlike most other genes, including mitochondrial, they evolve under neutral processes only and thus, phylogenetic relationships of orthologous pseudogenes are often excellent predictors of species phylogenies. The relationships defined by the $m \operatorname{stn} 1 a, m \operatorname{stn} 1 b$ and $m \operatorname{stn} 2 a$ trees may therefore by more representative of gene functional pressures rather than species phylogenies. Several lines of evidence nevertheless support a closer relationship for O. tshawytscha and $O$. nerka than previously suggested. This includes a unique $m \operatorname{stn} 2 b$ clade (Figure 8 ), strong support in the MSTN-1b, MSTN-2a and MSTN-2b amino acid trees (Additional files $1 \& 2$ ), and shared $m s t n 2 b$ indels (Figure 1). Revising salmonid phylogenetic relationships based solely on these data would clearly be premature. This is particularly true as the Splitstree data indicate a high level of hybridization or conflicting phylogenetic signal generated by other mechanisms within the family (Figures 10 \& 11). However, these studies do suggest the need to reassess the family, possibly by including more pseudogenes as well as more representatives of the myostatin gene family.

\section{Conclusions}

These studies together suggest that although the genomic organization of all paralogs is relatively well conserved, several notable structural differences that influence either coding sequences and/or transcript processing have indeed contributed to paralog divergence across taxa. Furthermore, the salmonid myostatin gene family appears to be actively diverging and is therefore a unique model system for investigating mechanisms that ultimately influence duplicate gene fate. Analysis of $m s t n 2 b$ structure in particular also suggests that a common ancestor to Oncorhynchus tshawytscha (a.k.a. king or chinook salmon) and O. nerka (a.k.a. sockeye salmon) diverged early and before the more recent salmonid radiation. This gene family is therefore a highly novel system for assessing gene and species phylogenies.

\section{Methods}

\section{Animals \& tissue handling}

Genomic (g)DNA or fin clips from different salmonids was provided by the Washington State University Aquaculture Core or by collaborators (see below). This includes samples from rainbow trout (Oncorhynchus mykiss), cutthroat trout (O. clarki), sockeye salmon (O. nerka), Chinook salmon (O. tshawytscha), coho salmon (O. kisutch), chum salmon (O. keta), Atlantic salmon (Salmo salar), brook trout (Salvelinus fontinalis), greyling (Thymallus thymallus; from Nicola Barson, University of Oslo), arctic greyling ( $T$. arcticus; from Christopher Myskiw, Fisheries \& Oceans Canada), lake and mountain whitefish (Coregonus clupeaformis \& Prosopium williamsoni; both from Peter Unmack, Brigham Young University), common whitefish (C. lavaretus) and common smelt (Retropinna retropinna; from Brendan Hicks, University of Waikato). Some sequences from $S$. salar and the Sv. fontinalis were downloaded from Genbank and accession numbers for all of the sequences used in this study, including novel sequences, are included in Table 5 . When fin clips were provided, gDNA was extracted by first incubating tissues in $3 \mathrm{ml}$ of lysis buffer (30 mM Tris, $8 \mathrm{M}$ Urea, 4\% w/v Chaps, $\mathrm{pH}$ 8.0) containing $20 \mathrm{mg} / \mathrm{ml}$ proteinase $\mathrm{K}$ at $60^{\circ} \mathrm{C}$. Three consecutive phenol:chloroform:isoamyl alcohol extractions were then performed and gDNA quality was verified on a $1 \%$ agarose gel. Fish were maintained in an AAALAC approved facility and samples were obtained according to animal use protocols preapproved by the universities' Animal Care and Use Committees.

\section{Gene cloning}

A multiple sequence alignment was first constructed using several known fish myostatin cDNA sequences. The consensus sequence was then used to generate PCR primers, some degenerate, suitable for amplifying partial or complete sequences of different myostatin genes. This 
Table 5 GenBank accession numbers

\begin{tabular}{|c|c|c|}
\hline Species & Gene & Accession number \\
\hline \multirow[t]{4}{*}{ O. mykiss } & mstnla & JN990743 \\
\hline & mstnib & JN990751 \\
\hline & mstn2a & JN990760 \\
\hline & $m s \operatorname{tn} 2 b$ & JN990770 \\
\hline \multirow[t]{6}{*}{ O. tshawytscha } & mstn1a (fall) & JN990744 \\
\hline & mstn1b (fall) & JN990754 \\
\hline & mstn1a (spring) & JN990745 \\
\hline & mstn1b (spring) & JN990755 \\
\hline & $m s \operatorname{tn} 2 a$ & JN990762 \\
\hline & $m s \operatorname{tn} 2 b$ & JN990772 \\
\hline \multirow[t]{2}{*}{ O. clarkii } & mstnla & JN990742 \\
\hline & mstn2a & JN990758 \\
\hline \multirow[t]{4}{*}{ O. kisutch } & mstnla & JN990737 \\
\hline & mstn1b & JN990738 \\
\hline & mstn2a & JN990759 \\
\hline & $m s \operatorname{tn} 2 b$ & JN990769 \\
\hline O. keta & $m s \operatorname{tn} 2 b$ & JN990768 \\
\hline \multirow[t]{3}{*}{ O. nerka } & mstn1b & JN990752 \\
\hline & mstn2a & JN990761 \\
\hline & $m s \operatorname{tn} 2 b$ & JN990771 \\
\hline \multirow[t]{4}{*}{ S. salar } & mstnla & EF392862.1/JN990746 \\
\hline & mstn1b & AJ316006.2/JN990753 \\
\hline & mstn2a & EF392863.1/JN990763 \\
\hline & $m s \operatorname{tn} 2 b$ & EF392864.1/JN990773 \\
\hline \multirow[t]{3}{*}{ Sv. Fontinalis } & mstnla & JN990748 \\
\hline & mstn1b & AY227655.1/JN990739 \\
\hline & $m s \operatorname{tn} 2 a$ & JN990764 \\
\hline \multirow[t]{3}{*}{ C. lavaretus } & mstnla & JN990741 \\
\hline & mstnib & JN990750 \\
\hline & mstn2a & JN990757 \\
\hline \multirow[t]{3}{*}{ C. clupeaformis } & mstnla & JN990740 \\
\hline & mstn1b & JN990749 \\
\hline & mstn2a & JN990756 \\
\hline T. arcticus & $m s t n 2 a$ & JN990765 \\
\hline T. thymallus & mstn2a & JN990766 \\
\hline P. williamsoni & mstn2a & JN990767 \\
\hline \multirow[t]{2}{*}{ R. retropinna } & mstn1 & JN990747 \\
\hline & mstn2 & JN990774 \\
\hline
\end{tabular}

C., Coregonus; O., Oncorhynchus; P., Prosopium; R., Retropinna; S., Salmo; SV., Salvelinus; $T$., Thymallus; fall/spring, seasonal runs.

includes primers specific to individual paralog subfamilies or primers that could presumably recognize conserved sites among all four genes. Many different primer sets were used and a list of primers will be supplied upon request. The specific PCR conditions varied depending upon the primer set used, although in general, $50 \mathrm{ng}$ gDNA was amplified using a high fidelity polymerase (Pfu, Stratagene, www.stratagene.com), primer-specific annealing temperatures and a 2 min extension period for each cycle. The PCR products were then sub-cloned into the Topo TA vector (Invitrogen, www.invitrogen.com) and sequenced in a university core.

\section{In silico analysis of gene structure}

Complementary DNA (cDNA) sequences for previously cloned MSTN-1a, $-1 \mathrm{~b},-2 \mathrm{a}$, and $-2 \mathrm{~b}$ genes were either downloaded from the National Center for Biotechnology Information (NCBI) or constructed from genomic DNA (gDNA) sequences. When gDNA was used, complete gene structures were identified using GenScan (http://genes. mit.edu/GENSCAN.html) and the exonic sequences were consequently used to construct coding sequences. Initial nucleic acid sequence analyses of cDNA were performed using ClustalW and default parameters in MacVector 10.0.2 (www.macvector.com). Coding sequences were similarly analyzed and both required manual editing. The intron splice site consensus sequences of the salmonid MSTN-2 genes were identified by searching for known splice site consensus sequences: 5' (A or C)AG/GURAGU where $\mathrm{R}=\mathrm{G}$ or A and 3' CAG/G. Putative branch points, also necessary for mRNA splicing, were determined by searching for TNCTRAY where $\mathrm{N}$ = any nucleotide, $\mathrm{R}=$ $\mathrm{G}$ or $\mathrm{A}$, and $\mathrm{Y}=\mathrm{C}$ or $\mathrm{T}$. The number and location of YCAY motifs, regions known to influence spliceosome binding, were also located.

\section{Phylogenetic analyses}

Analyses were performed using cDNA or gDNA collected from 14 different salmonid species and with the common smelt as the outgroup. Sequences were organized into separate files containing MSTN-1 or MSTN-2 genes ( $\mathrm{a} \& \mathrm{~b}$ paralogs in each) and aligned using MAFFT [43] before testing for optimal substitution using JModelTest [44], all of which fit a General Time Reversible (GTR) model with an estimated gamma distribution. Phylogenetic trees were constructed using a Maximum Likelihood (ML) method and a Bayesian method through the programs PhyML $3.0[45,46]$ and MrBayes 3.2 [47], respectively. The accuracy of the resulting topologies was determined through a non-parametric analysis of 1,000 bootstraps or posterior probabilities after 10,000,000 generations [48].

To confirm the systematics of the salmonid lineages, a second phylogenetic analysis was performed using amino acid sequences. Intronic sequences were first located using GENSCAN [49] and GeneMark-E [50] and excised before aligning the resulting sequences with MAFFT and testing for the optimal substitution model with ProtTest3 [51], which resulted in a Jones, Taylor, Thornton (JTT) matrix with an estimated gamma distribution. Phylogenetic trees were then constructed using PhyML 3.0 with a 1,000 
bootstrap analysis MrBayes 3.2 with 10,000,000 generations, sampling every 1,000 generations.

Evidence of positive selection was assessed by analysis of dN/dS ratios using Phylogenetic Analysis by Maximum Likelihood (PAML) [52]. Amino acid alignments were coordinated into codons using PAL2NAL [53] in order to estimate $\omega$. Free- and single-ratio estimations of $\omega$ were conducted along every branch and the results were compared using a chi-squared analysis to determine evidence for supporting a free-ratios model, which only occurred in the MSTN-2 tree. A phylogenetic tree was then constructed to indicate the $\mathrm{dN} / \mathrm{dS}$ values for each branch. Values greater than 1 demonstrate branches with high rate variation and thus, possible signals for positive selection. An additional analysis, testing the branch-site model A of PAML, was also performed. Using this model, $\omega$ is estimated on a foreground branch against the background branches of the tree to determine rate variation. The results were compared using a $\chi^{2}$ likelihood ratio test to determine if there was sufficient evidence of supporting the alternative model of an independent $\omega$ value for the foreground branch from the rest of the tree over the null model of a fixed $\omega$ value of 1 throughout each branch of the tree. A Bayes Empirical Bayes (BEB) analysis was also performed, searching for potential sites within the alignment that were under positive selection. A false discovery rate (FDR) test was performed and the R-based program QVALUE [54] was used to estimate the proportion of true null hypotheses. The program was run using the default settings with a preset FDR of 5\%. To further test the occurrence and amount of hybridization and/or incongruent phylogenetic signal that might have occurred between salmonids, a splitstree network was constructed using all four paralogs and the program SplitsTree4 [55].

\section{Additional files}

Additional file 1: MSTN-1a, -1b amino acid phylogeny. This phylogeny was created using an amino acid alignment of MSTN-1a and 1b. Introns were excised from the amino acid sequences by the programs GENSCAN and GeneMark-E. The alignments were then made using MAFFT, and the phylogeny was constructed through PhyML 3.0 $[45,46]$, with a 1000 bootstrap analysis.

Additional file 2: MSTN-2a, $-2 b$ amino acid phylogeny. This phylogeny was created using an amino acid alignment of MSTN-2a and 2b. Introns were excised from the amino acid sequences by the programs GENSCAN and GeneMark-E. The alignments were then made using MAFFT, and the phylogeny was constructed through PhyML 3.0 [45,46], with a 1000 bootstrap anlaysis.

Additional file 3: (A) Bayesian Phylogeny of Myostatin proteins (MSTN-1a/1b and MSTN-2a/2b). The topology was generated using all available myostatin proteins from all for myostatin groups MSTN-1a/1b, and MSTN-2a/2b. The phylogeny was constructed using MrBayes 3.2 [47] with posterior probabilities indicated on the internal nodes of the tree. The tree was rooted with $R$. retropinna as the outgroup. (B) Maximum Likelihood Phylogeny of Myostatin proteins (MSTN-1a/1b and MSTN-2a/ 2b). The topology was generated using all available myostatin proteins from all for myostatin groups MSTN-1a/1b, and MSTN-2a/2b. The phylogeny was constructed using Phyml $3.0[45,46]$ with 1,000 bootstraps indicated on the internal nodes of the tree. The tree was rooted with $R$. retropinna as the outgroup. (C) Bayesian Phylogeny of Myostatin genes ( $m s \operatorname{tn} 1 a / 1 b$ and $m s t n 2 a / 2 b$ ). The topology was generated using all available myostatin genes from all for myostatin groups mstn $1 a / 1 b$, and mstn2a/2b. The phylogeny was constructed using MrBayes 3.2 [47] with posterior probabilities indicated on the internal nodes of the tree. The tree was rooted with $R$. retropinna as the outgroup.

Additional file 4: BEB analysis of MSTN-1a/1b and MSTN-2a/2b. The amino acid letter represents the amino acid for that position in the first sequence of the alignment used to for the analysis (O. kisutch for MSTN- $1 \mathrm{a} / 1 \mathrm{~b}$ and C. clupeaformis for MSTN-2a/2b). An * indicates there was a gap in the alignment at that position for the first sequence in the alignment. Only sites with probabilities greater than $95 \%$ are listed.

\section{Competing interests}

DAL is a current section editor for this journal. All of the other authors have nothing to disclose and assert no competing or potential conflicts of interests.

\section{Authors' contributions}

Experiments were performed by CBL, TN, RAH and VB-V while MFJ and DKG trained CBL and TN and assisted in cloning. The manuscript was written primarily by CBL and TN with editorial assistance from DAL and BDR The latter also planned and supervised these studies and interpreted the data. All authors read and approved the final manuscript.

\section{Acknowledgements}

This project was supported by grants from the National Science Foundation to Buel D. Rodgers (0840644) and David A. Liberles (0743374).

\section{Author details}

'Department of Animal Sciences, Washington State University, Pullman, WA 99164, USA. ${ }^{2}$ School of Molecular Biosciences, Washington State University, Pullman, WA 99164, USA. 'Washington Center for Muscle Biology, Washington State University, Pullman, WA 9916, USA. ${ }^{4}$ Department of Molecular Biology, University of Wyoming, Laramie, WY 82071, USA.

Received: 27 June 2012 Accepted: 22 August 2012

Published: 8 October 2012

\section{References}

1. Rodgers BD, Garikipati DK: Clinical, agricultural, and evolutionary biology of myostatin: a comparative review. Endocr Rev 2008, 29(5):513-534.

2. Patel $\mathrm{K}$, Macharia R, Amthor $\mathrm{H}$ : Molecular mechanisms involving IGF-1 and myostatin to induce muscle hypertrophy as a therapeutic strategy for Duchenne muscular dystrophy. Acta Myol 2005, 24(3):230-241.

3. Bossola M, Pacelli F, Tortorelli A, Rosa F, Doglietto GB: Skeletal muscle in cancer cachexia: the ideal target of drug therapy. Curr Cancer Drug Targets 2008, 8(4):285-298.

4. Mak RH, Rotwein P: Myostatin and insulin-like growth factors in uremic sarcopenia: the yin and yang in muscle mass regulation. Kidney Int 2006, 70(3):410-412

5. Williams NG, Interlichia JP, Jackson MF, Hwang D, Cohen P, Rodgers BD: Endocrine actions of myostatin: systemic regulation of the IGF and IGF binding protein axis. Endocrinology 2011, 152(1):172-180.

6. Lakshman KM, Bhasin S, Corcoran C, Collins-Racie LA, Tchistiakova L, Forlow SB, St Ledger K, Burczynski ME, Dorner AJ, Lavallie ER: Measurement of myostatin concentrations in human serum: circulating concentrations in young and older men and effects of testosterone administration. $\mathrm{Mol}$ Cell Endocrinol 2009, 302(1):26-32.

7. Langley B, Thomas M, Bishop A, Sharma M, Gilmour S, Kambadur R: Myostatin inhibits myoblast differentiation by down regulating MyoD expression. J Biol Chem 2002, 277:49831-49840.

8. Rios R, Carneiro I, Arce VM, Devesa J: Myostatin is an inhibitor of myogenic differentiation. Am J Physiol Cell Physiol 2002, 282(5):C993-C999. 
9. Joulia D, Bernardi H, Garandel V, Rabenoelina F, Vernus B, Cabello G: Mechanisms involved in the inhibition of myoblast proliferation and differentiation by myostatin. Exp Cell Res 2003, 286(2):263-275.

10. Garikipati DK, Rodgers BD: Myostatin inhibits myosatellite cell proliferation and consequently activates differentiation: evidence for endocrineregulated transcript processing. J Endocrinol 2012, 215(1):177-187.

11. Garikipati DK, Rodgers BD: Myostatin stimulates myosatellite cell differentiation in a novel model system: evidence for gene subfunctionalization. AJP Regula Int Comp Phys 2012, 302(9):R1059-R1066.

12. Kerr T, Roalson EH, Rodgers BD: Phylogenetic analysis of the myostatin gene sub-family and the differential expression of a novel member in zebrafish. Evol Dev 2005, 7(5):390-400.

13. Rodgers BD, Roalson EH, Weber GM, Roberts SB, Goetz FW: A proposed nomenclature consensus for the myostatin gene family. Am J Physiol Endocrinol Metab 2007, 292(2):E371-E372.

14. Amores A, Force A, Yan YL, Joly L, Amemiya C, Fritz A, Ho RK, Langeland J, Prince $V$, Wang $Y L$, et al: Zebrafish hox clusters and vertebrate genome evolution. Science 1998, 282(5394):1711-1714.

15. Postlethwait JH, Yan YL, Gates MA, Horne S, Amores A, Brownlie A Donovan A, Egan ES, Force A, Gong Z, et al: Vertebrate genome evolution and the zebrafish gene map. Nat Genet 1998, 18(4):345-349.

16. Garikipati DK, Gahr SA, Roalson EH, Rodgers BD: Characterization of rainbow trout myostatin-2 genes (rtMSTN-2a and -2b): genomic organization, differential expression, and pseudogenization. Endocrinology 2007, 148(5):2106-2115.

17. Innan $\mathrm{H}$, Kondrashov F: The evolution of gene duplications: classifying and distinguishing between models. Nat Rev Genet 2010, 11(2):97-108.

18. Tellgren A, Berglund AC, Savolainen P, Janis CM, Liberles DA: Myostatin rapid sequence evolution in ruminants predates domestication. $\mathrm{Mol}$ Phylogenet Evol 2004, 33(3):782-790.

19. Ke S, Zhang XH, Chasin LA: Positive selection acting on splicing motifs reflects compensatory evolution. Genome Res 2008, 18(4):533-543.

20. Plass M, Eyras E: Differentiated evolutionary rates in alternative exons and the implications for splicing regulation. BMC Evol Biol 2006, 6:50.

21. Xing $Y$, Lee $C$ : Alternative splicing and RNA selection pressureevolutionary consequences for eukaryotic genomes. Nat Rev Genet 2006, 7(7):499-509.

22. Ostbye TK, Wetten OF, Tooming-Klunderud A, Jakobsen KS, Yafe A, Etzioni S, Moen T, Andersen O: Myostatin (MSTN) gene duplications in Atlantic salmon (Salmo salar): evidence for different selective pressure on teleost MSTN-1 and -2. Gene 2007, 403(1-2):159-169.

23. Koop BF, von Schalburg KR, Leong J, Walker N, Lieph R, Cooper GA, Robb A, Beetz-Sargent M, Holt RA, Moore R, et al: A salmonid EST genomic study: genes, duplications, phylogeny and microarrays. BMC Genomics 2008, 9:545

24. Hughes T, Liberles DA: Whole-genome duplications in the ancestral vertebrate are detectable in the distribution of gene family sizes of tetrapod species. J Mol Evol 2008, 67(4):343-357.

25. Hughes T, Liberles DA: The power-law distribution of gene family size is driven by the pseudogenisation rate's heterogeneity between gene families. Gene 2008, 414(1-2):85-94.

26. He X, Zhang J: Gene complexity and gene duplicability. Curr Biol 2005, 15(11):1016-1021.

27. He X, Zhang J: Rapid subfunctionalization accompanied by prolonged and substantial neofunctionalization in duplicate gene evolution. Genetics 2005, 169(2):1157-1164.

28. Rastogi S, Liberles DA: Subfunctionalization of duplicated genes as a transition state to neofunctionalization. BMC Evol Biol 2005, 5:28.

29. Altschmied J, Delfgaauw J, Wilde B, Duschl J, Bouneau L, Volff JN, Schartl M: Subfunctionalization of duplicate mitf genes associated with differential degeneration of alternative exons in fish. Genetics 2002, 161(1):259-267.

30. Cusack BP, Wolfe KH: When gene marriages don't work out: divorce by subfunctionalization. Trends Genet 2007, 23(6):270-272.

31. Su Z, Wang J, Yu J, Huang X, Gu X: Evolution of alternative splicing after gene duplication. Genome Res 2006, 16(2):182-189.

32. Morissette MR, Cook SA, Foo S, McKoy G, Ashida N, Novikov M, ScherrerCrosbie M, Li L, Matsui T, Brooks G, et al: Myostatin regulates cardiomyocyte growth through modulation of Akt signaling. Circ Res 2006, 99(1):15-24.

33. Trendelenburg AU, Meyer A, Rohner D, Boyle J, Hatakeyama S, Glass DJ: Myostatin reduces Akt/TORC1/p70S6K signaling, inhibiting myoblast differentiation and myotube size. Am J Physiol Cell Physiol 2009, 296(6):C1258-C1270.

34. Amirouche A, Durieux AC, Banzet S, Koulmann N, Bonnefoy R, Mouret C, Bigard X, Peinnequin A, Freyssenet D: Down-regulation of Akt/mammalian target of rapamycin signaling pathway in response to myostatin overexpression in skeletal muscle. Endocrinology 2009, 150(1):286-294.

35. Frost RA, Lang CH: Protein kinase B/Akt: a nexus of growth factor and cytokine signaling in determining muscle mass. J Appl Physiol 2007, 103(1):378-387.

36. Solomon AM, Bouloux PM: Modifying muscle mass - the endocrine perspective. J Endocrinol 2006, 191(2):349-360.

37. Interlichia JP, Williams NG, Rodgers BD: A Rapid, Valid and Inexpensive Assay for Measuring Epiphyseal Plates in Mouse Tibia. GH IGF Res 2009, in press.

38. Shyu KG, Ko WH, Yang WS, Wang BW, Kuan P: Insulin-like growth factor-1 mediates stretch-induced upregulation of myostatin expression in neonatal rat cardiomyocytes. Cardiovasc Res 2005, 68(3):405-414.

39. Morissette MR, Cook SA, Buranasombati C, Rosenberg MA, Rosenzweig A: Myostatin inhibits IGF-l-induced myotube hypertrophy through Akt. Am J Physiol Cell Physiol 2009, 297(5):C1124-C1132.

40. Dredge BK, Darnell RB: Nova regulates $G A B A(A)$ receptor gamma2 alternative splicing via a distal downstream UCAU-rich intronic splicing enhancer. Mol Cell Biol 2003, 23(13):4687-4700.

41. Dredge BK, Stefani G, Engelhard CC, Darnell RB: Nova autoregulation reveals dual functions in neuronal splicing. EMBO J 2005, 24(8):1608-1620.

42. Jensen KB, Dredge BK, Stefani G, Zhong R, Buckanovich RJ, Okano HJ, Yang $Y Y$, Darnell RB: Nova-1 regulates neuron-specific alternative splicing and is essential for neuronal viability. Neuron 2000, 25(2):359-371.

43. Katoh K, Kuma K, Toh H, Miyata T: MAFFT version 5: improvement in accuracy of multiple sequence alignment. Nucleic Acids Res 2005 33(2):511-518.

44. Posada D: jModelTest: phylogenetic model averaging. Mol Biol Evol 2008 , 25(7):1253-1256

45. Guindon S, Gascuel O: A simple, fast, and accurate algorithm to estimate large phylogenies by maximum likelihood. Syst Biol 2003, 52(5):696-704.

46. Guindon S, Dufayard JF, Lefort V, Anisimova M, Hordijk W, Gascuel O: New Algorithms and Methods to Estimate Maximum-Likelihood Phylogenies: Assessing the Performance of PhyML 3.0. Syst Biol 2010, 59(3):307-321.

47. Ronquist F, Teslenko M, van der Mark P, Ayres DL, Darling A, Hohna S, Larget B, Liu L, Suchard MA, Huelsenbeck JP: MrBayes 3.2: efficient Bayesian phylogenetic inference and model choice across a large model space. Syst Biol 2012, 61(3):539-542.

48. Felsenstein J: Confidence-Limits on Phylogenies with a Molecular Clock. Syst Zool 1985, 34(2):152-161

49. Burge C, Karlin S: Prediction of complete gene structures in human genomic DNA. J Mol Biol 1997, 268(1):78-94.

50. Lomsadze A, Ter-Hovhannisyan V, Chernoff YO, Borodovsky M: Gene identification in novel eukaryotic genomes by self-training algorithm. Nucleic Acids Res 2005, 33(20):6494-6506.

51. Darriba D, Taboada GL, Doallo R, Posada D: ProtTest 3: fast selection of best-fit models of protein evolution. Bioinformatics 2011, 27(8):1164-1165.

52. Yang Z: PAML 4: phylogenetic analysis by maximum likelihood. $\mathrm{Mol}$ Biol Evol 2007, 24(8):1586-1591.

53. Suyama M, Torrents D, Bork P: PAL2NAL: robust conversion of protein sequence alignments into the corresponding codon alignments. Nucleic Acids Res 2006, 34(Web Server issue):W609-W612.

54. Dalmasso $C$, Broet $P$, Moreau T: A simple procedure for estimating the false discovery rate. Bioinformatics 2005, 21(5):660-668.

55. Huson DH, Bryant D: Application of phylogenetic networks in evolutionary studies. Mol Biol Evol 2006, 23(2):254-267.

doi:10.1186/1471-2148-12-202

Cite this article as: Lawson et al:: The salmonid myostatin gene family: a novel model for investigating mechanisms that influence duplicate gene fate. BMC Evolutionary Biology 2012 12:202 